\title{
Eating Behaviour Changes during the COVID-19 Pandemic: A Systematic Review of Longitudinal Studies
}

\author{
Cristina González-Monroy (D), Irene Gómez-Gómez, Cristian M. Olarte-Sánchez (D) and Emma Motrico *iD \\ Department of Psychology, Universidad Loyola Andalucía, Dos Hermanas, 41704 Seville, Spain; \\ cgonzalezmonrroy@al.uloyola.es (C.G.-M.); igomezg@uloyola.es (I.G.-G.); cmolarte@uloyola.es (C.M.O.-S.) \\ * Correspondence: emotrico@uloyola.es
}

check for

updates

Citation: González-Monroy, C.; Gómez-Gómez, I.; Olarte-Sánchez, C.M.; Motrico, E. Eating Behaviour Changes during the COVID-19

Pandemic: A Systematic Review of Longitudinal Studies. Int. J. Environ Res. Public Health 2021, 18, 11130. https://doi.org/10.3390/ ijerph182111130

Academic Editors: Thomas E. Dorner and Katharina Viktoria Stein

Received: 18 June 2021

Accepted: 20 October 2021

Published: 22 October 2021

Publisher's Note: MDPI stays neutral with regard to jurisdictional claims in published maps and institutional affiliations.

Copyright: (c) 2021 by the authors. Licensee MDPI, Basel, Switzerland. This article is an open access article distributed under the terms and conditions of the Creative Commons Attribution (CC BY) license (https:// creativecommons.org/licenses/by/ $4.0 /)$.

\begin{abstract}
Eating behaviour is a complex construct that is liable to be modified by external factors. Due to the outbreak of coronavirus disease 2019 (COVID-19), many restrictive measures were carried out with the aim of reducing the impact of this disease. As a result, lifestyles were disrupted, which could affect eating behaviours. The aim of this systematic review of longitudinal studies was to assess changes in eating behaviour during the COVID-19 pandemic by establishing a comparison of eating behaviours before and after the outbreak of the pandemic. This study followed the PRISMA guidelines (PROSPERO: CRD42020203246), whereas to assess the quality of the studies, the Newcastle-Ottawa Quality Assessment Scale (NOS) was applied. Out of a set of 826 studies, 23 were included in this systematic review. The main findings provided information about a shift towards modified eating behaviours, characterized by an increased snack frequency and a preference for sweets and ultra-processed food rather than fruits, vegetables, and fresh food. Additionally, an increased alcohol consumption was found among different countries. Consequently, adherence to healthy diets decreased. These findings are relevant to future policies and strategies to assess nutrition in cases of alarming situations such as the current COVID-19 pandemic.
\end{abstract}

Keywords: eating behaviour; COVID-19; systematic review; longitudinal studies

\section{Introduction}

The appearance of coronavirus disease 2019 (COVID-19), an infectious disease caused by a coronavirus [1], has led to serious changes worldwide from late 2019 to the present day. Not only relationship patterns but also lifestyle habits have changed due to lockdown conditions, social distancing, reduced capacity in enclosed spaces and curfews proposed by governments. Although the aim of this measure was to fight the spread of the pandemic, the impact on daily life has been immense [2,3]. Since the first wave of the COVID-19 pandemic, many governments have ordered their population to stay at home while following security measures. As a result, people from many countries had to eat all of their meals at home while adapting their physical activity indoors due to not being allowed to go to public places or even to the gym [4,5]. In other cases, lifestyle habits suffered abrupt changes due to a lack of social services such as school cantines [6], and food insecurity [7]. As a result, eating behaviours could change across age groups [8-10].

Eating behaviours may be defined as food choices that are moderated by consumption trends, personal preferences, specific diets and calorie counting [11,12]. Regarding this definition, some cross-sectional studies have been carried out since the outbreak of the pandemic with the aim of achieving this objective [8-10,13-17]. However, as cross-sectional studies cannot identify differences in eating behaviour in comparison to previous baselines before the pandemic, bias can appear when trying to interpret the results [18-22]. This limitation appears to have been solved by summarizing the information in a few systematic reviews that have been carried out with the aim of exploring the impact of the pandemic on weight-related behaviours that include not only eating behaviours but also politics 
affecting the food supply and the health consequences in terms of nutritional status [18-22]. In this sense, a wide range of eating patterns that include both food restriction and loss of control over eating have been found, especially when analysing unhealthy food consumption $[19,20]$. This information remains relevant for people without comorbidities but also for vulnerable groups such as the older population and people with obesity that have experienced more acute changes in their eating behaviour [21,22].

Specifically, it has been found that the COVID-19 pandemic has had a global impact on daily diet among adults [19]. Although it has not been possible to establish a correlation between weight gain and changes on eating behaviour, an increased appetite accompanied by a higher consumption of snacks and a greater number of daily meals have been found. In the case of children, results appear to point at similar conclusions as the closure of school canteens has affected many families [6]. This situation has worsened the quality of diet in families with little resources as well as in families in which all members were workers. Finally, regarding dependent persons such as the elderly, people with morbid obesity and people with other type of disabilities, scientific evidence has linked preventive measures of physical contact restriction to changes in eating behaviour. Those changes have been associated to a significant decline in health nutrition [20].

This panorama has been worse in developing countries, where the outbreak of the COVID-19 pandemic has enhanced the need of proper define 'food security'. Although apparently there was 'availability' and 'accessibility' to food supplies, many developing countries had no adequate supply chains. Consequently, families were not properly supplied with food, so they had to change their eating behaviours, which, in turn, contributed to a decline in health nutrition [7]. It is important to establish this dichotomy when talking about 'eating behaviour changes during the COVID-19 pandemic' because decision making about food may be motivated by different reasons, although the outbreak of the COVID-19 pandemic primarily triggered those changes.

In light of the above, the results suggest the need to properly understand the changes in eating behaviour that occurred during the development of the COVID-19 pandemic by focusing on longitudinal studies that can address changes by establishing comparisons before and after the outbreak of the pandemic. Therefore, the main objective of this study was to undertake a systematic review from September 2019 to July 2021 to evaluate eating behaviour changes, taking into account the following research question: which changes have occurred in eating behaviour during the COVID-19 pandemic? It is expected to produce an accurate description of those changes to better understand their consequences. These findings remain relevant to future policies and strategies to assess nutrition in cases of alarming situations such as the current COVID-19 pandemic.

\section{Materials and Methods}

This review followed the Preferred Reporting Items for Systematic Reviews and MetaAnalyses (PRISMA) guidelines [23]. Additionally, it was registered in the International Prospective Register of Systematic Reviews database (PROSPERO) (registration number CRD42020203246).

\subsection{Search Strategy}

The search strategy was defined by establishing the research question as well as the inclusion and exclusion criteria according to the PICO (Population/Participants, Intervention, Comparison and Outcomes) model [24], as shown in Table 1. 
Table 1. Inclusion and Exclusion Criteria.

\begin{tabular}{|c|c|c|}
\hline & Inclusion Criteria & Exclusion Criteria \\
\hline Participants & All participants & None \\
\hline Outcome & $\begin{array}{l}\text { Eating behaviour changes during the COVID-19 } \\
\text { pandemic. }\end{array}$ & $\begin{array}{l}\text { Those studies which do not assess } \\
\text { pre/post-records of data among participants. }\end{array}$ \\
\hline Type of study & $\begin{array}{l}\text { Longitudinal studies (prospective and } \\
\text { retrospective) }\end{array}$ & $\begin{array}{l}\text { Cross-sectional studies, reviews, letters to the } \\
\text { editor, recommendations. }\end{array}$ \\
\hline Language & All languages & None \\
\hline Setting & All settings & None \\
\hline
\end{tabular}

The search was first piloted in the PubMed database on 27 July 2021 (as shown in Table 2) and filtered since 31 December 2019. Then, it was replicated in three other databases: Cochrane Library, PsycInfo and Web of Science. Then, two clusters of search terms were designed. The first included terms related to COVID-19, while the second was related to eating behaviour changes. To develop a representative advanced search, the selected words were chosen from the Medical Subject Headings (MeSH) thesaurus. Additionally, lists of references from previous studies that were selected as representative of this review were examined.

Table 2. Search Strategy in PubMed.

\begin{tabular}{|c|}
\hline \\
\hline $\begin{array}{l}\text { Search Strategy } \\
\left(\left(\left(\left(\left(()^{\prime C} \mathrm{COVID}-19^{\prime \prime}\right.\right.\right.\right.\right.\end{array}$ \\
\hline Terms]) OR “coronavirus pandemic"[Title/Abstract]") OR 2019-nCov"[Title/Abstract]) OR \\
\hline “confinement”[Title/Abstract]) OR “lockdown”[Title/Abstract]) AND ((((((((“feeding \\
\hline behavior"[MeSH Terms]) OR “diet, healthy"[MeSH Terms]) OR “diet*” [MeSH Terms]) “Diet, \\
\hline Food, and Nutrition"[MeSH Terms]) OR “healthy eating"[Title/Abstract]) OR \\
\hline “diet" ${ }^{*}$ [Title/Abstract]) OR “dietary pattern"[Title/Abstract]) OR “food intake"[Title/Abstract]) \\
\hline OR “nutrit*”[Title/Abstract]) OR “diet change"[Title/Abstract] AND ((cohort [Title/Abstract]) \\
\hline OR (longitudinal [Title/Abstract]) OR (follow-up [Title/Abstract]) OR (prospective \\
\hline [Title/Abstract]) OR (retrospective [Title/Abstract]) OR (observational [Title/Abstract])) \\
\hline
\end{tabular}

\subsection{Study Selection Process}

This systematic review comprised 3 main steps. First, duplicated content was identified and deleted. Second, by following the inclusion/exclusion criteria, the studies were reviewed by title/abstract. Finally, those that passed the screening were reviewed by a full-text reading. All of these steps were conducted independently by two reviewers, and in cases of disagreement, a third reviewer was assigned to resolve any discrepancies. The inter-agreement of the total selection between reviewers was almost perfect [25] (Cohen Kappa Index $=0.85 ; 95 \%$ CI, 0.49 to 1.00 ).

\section{Results}

\subsection{Study Selection}

The search strategy led to a set of 816 studies. In addition, 10 studies were identified after examining the lists of references from the representative studies. Consequently, the first stage of the revision gathered 826 studies. Of those, 106 studies met the inclusion criteria under the title/abstract review. After a full-text reading, 83 studies were removed due to not meeting the inclusion criteria. Thus, 23 studies passed the screening process, which led to the next phase. The screening process is summarized in Figure 1. 
Identification of studies via databases and registers

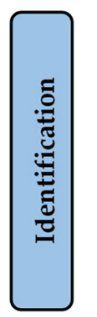

\begin{tabular}{|c|c|}
\hline $\begin{array}{c}\text { Records identified from: } \\
\text { Databases }(n=816) \\
\text { PubMed }=381 \\
\text { Cochrane Library }=286 \\
\text { PsycInfo }=73 \\
\text { Web of Science }=76\end{array} \quad \begin{array}{l}\begin{array}{r}\text { Records removed before screening: } \\
\text { Duplicate records removed } \\
(n=86)\end{array} \\
\text { Registers }(n=10)\end{array} \quad \begin{array}{l}\text { Records marked as ineligible } \\
\text { by automation tools }(n=0) \\
\text { Records removed for other } \\
\text { reasons }(n=0)\end{array}$ \\
\hline
\end{tabular}

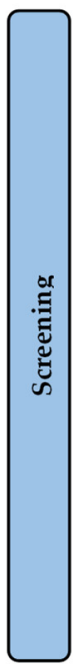
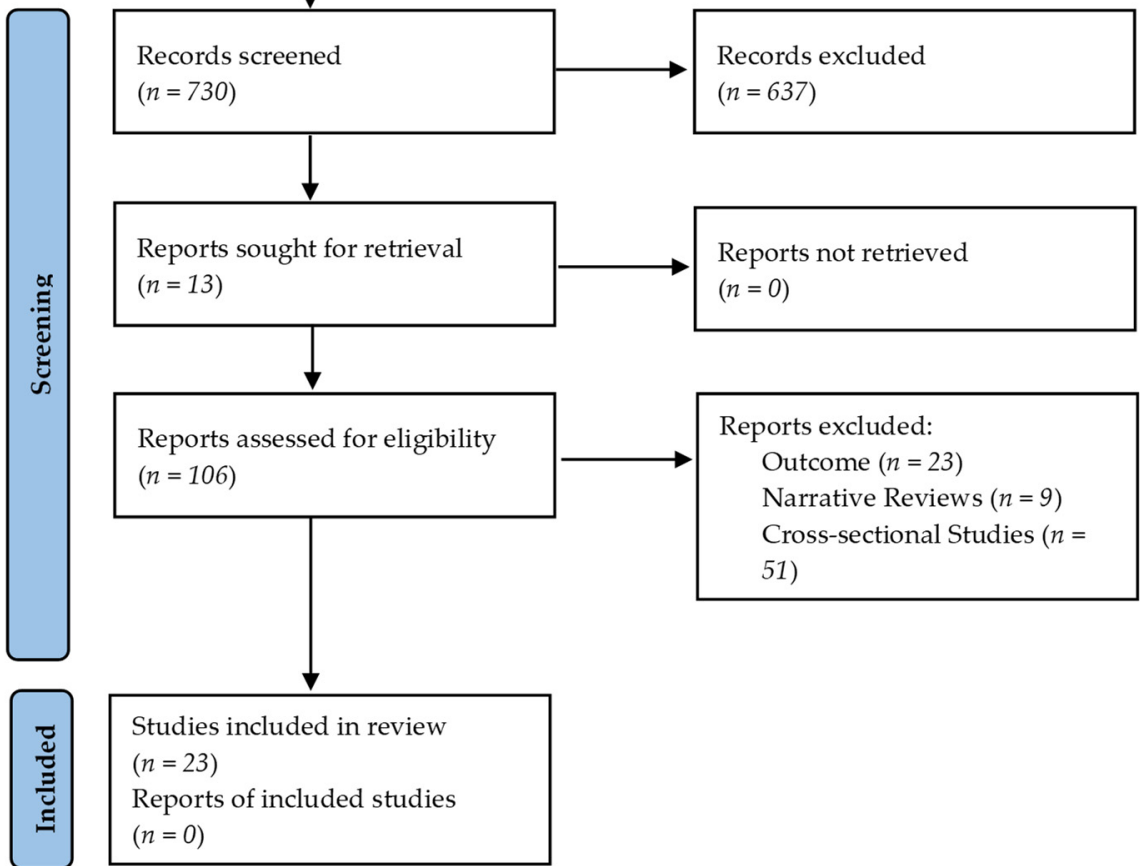

$$
\begin{aligned}
& \text { Studies included in review } \\
& (n=23) \\
& \text { Reports of included studies } \\
& (n=0)
\end{aligned}
$$

Figure 1. PRISMA flow diagram of the included and excluded studies throughout the systematic review protocol.

\subsection{Study Characteristics}

The characteristics of the selected studies are summarized in Table 3. The variables are taken for in-depth analysis from the paragraphs below. 
Table 3. Characteristics of the included studies.

\begin{tabular}{|c|c|c|c|c|c|c|c|c|c|c|}
\hline $\begin{array}{c}\text { First } \\
\text { Author/Year/ } \\
\text { Region } \\
\text { (Country) }\end{array}$ & $\begin{array}{l}\text { Design } \\
\text { (Study } \\
\text { Type) }\end{array}$ & $\begin{array}{l}\text { Length of } \\
\text { Follow-Up } \\
\text { (Weeks) }\end{array}$ & $\begin{array}{l}\text { Target } \\
\text { Population } \\
\text { (Age) }\end{array}$ & Inclusion Criteria & Exclusion Criteria & $\begin{array}{l}\text { Sample Size at } \\
\text { Baseline (Total } \\
n / \text { Female }(\%))\end{array}$ & Assessment Tool & $\begin{array}{c}\text { Target } \\
\text { Lifestyles }\end{array}$ & $\begin{array}{c}\text { Sample Size at } \\
\text { Follow-Up } \\
\text { (Total } n / \text { Female } \\
(\%))\end{array}$ & $\begin{array}{l}\text { Statistical } \\
\text { Technique/s }\end{array}$ \\
\hline $\begin{array}{l}\text { Khare, (2020) } \\
\text { Bhopal } \\
\text { (India) }\end{array}$ & PS & 3 weeks & $\begin{array}{l}\text { Patients aged } \\
\text { 18-65 years old } \\
\text { with a diagnose } \\
\text { of type } 2 \\
\text { diabetes } \\
\text { mellitus }\end{array}$ & $\begin{array}{l}\text { Adult patients with } \\
\text { previously } \\
\text { diagnosed type } 2 \\
\text { diabetes mellitus on } \\
\text { treatment, and } \\
\text { willing to participate } \\
\text { in the study }\end{array}$ & $\begin{array}{l}\text { Sick patient, patients } \\
\text { with established } \\
\text { microvascular or } \\
\text { macrovascular } \\
\text { complication of } \\
\text { diabetes, pregnancy, } \\
\text { and not willing to } \\
\text { participate in the } \\
\text { study }\end{array}$ & $\begin{array}{c}n=143 \\
\text { Female }=36.36 \% \\
(n=52)\end{array}$ & $\begin{array}{l}\text { Questionnaire } \\
\text { designed for this } \\
\text { study }\end{array}$ & $\begin{array}{l}\text { Diet, exercise, } \\
\text { sleep, } \\
\text { glycaemic } \\
\text { control }\end{array}$ & $\begin{array}{c}n=143 \\
\text { Female = } \\
36.36 \%(n=52)\end{array}$ & $\begin{array}{l}\text { Descriptive analysis } \\
\text { (paired } t \text {-test) }\end{array}$ \\
\hline $\begin{array}{l}\text { Munasinghe, } \\
\text { (2020) } \\
\text { New South } \\
\text { Wales } \\
\text { (Australia) }\end{array}$ & PS & 22 weeks & $\begin{array}{l}\text { Adolescents } \\
\text { aged 13-19 } \\
\text { years of a } \\
\text { Sydney } \\
\text { population } \\
\text { catchment }\end{array}$ & $\begin{array}{l}\text { (1) Age 13-19 years } \\
\text { old (2) living in } \\
\text { Sidney }\end{array}$ & No & $\begin{array}{c}\quad n=1298 \\
\text { Female }=80.5 \% \\
\quad(n=1045)\end{array}$ & $\begin{array}{l}\text { Self-reported dietary } \\
\text { behaviour was } \\
\text { measured using } \\
\text { questions validated } \\
\text { for adolescents by } \\
\text { the NSW Centre for } \\
\text { Public Health } \\
\text { Nutrition }\end{array}$ & $\begin{array}{l}\text { Physical } \\
\text { activity, } \\
\text { sedentary } \\
\text { behaviour, } \\
\text { dietary } \\
\text { behaviour, } \\
\text { screen time }\end{array}$ & $\begin{array}{c}n=582 \\
\text { Female }= \\
79.89 \%(n=465)\end{array}$ & $\begin{array}{l}\text { Multivariate } \\
\text { multilevel } \\
\text { mixed-effect logistic } \\
\text { regression models }\end{array}$ \\
\hline $\begin{array}{c}\text { Pietrobelli, } \\
\text { (2020) } \\
\text { Verona (Italy) }\end{array}$ & RS & 11 weeks & $\begin{array}{l}\text { Children and } \\
\text { adolescents } \\
\text { aged 6-18 } \\
\text { years old }\end{array}$ & $\begin{array}{l}\text { Children and } \\
\text { adolescents with } \\
\text { overweight and } \\
\text { obesity }\end{array}$ & No & $\begin{array}{c}n=41 \\
\text { Female }=46.34 \% \\
\quad(n=19)\end{array}$ & $\begin{array}{l}12 \text { items lifestyle } \\
\text { questionnaire } \\
\text { (sports activity } \\
\text { participation, screen } \\
\text { time, sleep } \\
\text { behaviour, and } \\
\text { eating habits, } \\
\text { focusing on servings } \\
\text { of red meat, pasta, } \\
\text { snacks, fruits, and } \\
\text { vegetables) }\end{array}$ & $\begin{array}{l}\text { Physical } \\
\text { activity, screen } \\
\text { time, sleep } \\
\text { behaviour and } \\
\text { eating habits }\end{array}$ & $\begin{array}{c}n=41 \\
\text { Female }= \\
46.34 \%(n=19)\end{array}$ & $\begin{array}{c}\text { (1) Descriptive } \\
\text { statistics of the } \\
\text { participant's } \\
\text { baseline, (2) paired } \\
t \text {-tests, (3) } \\
\text { correlation analysis, } \\
\text { (4) independent } \\
\text { two-sample } t \text {-tests }\end{array}$ \\
\hline
\end{tabular}


Table 3. Cont.

\begin{tabular}{|c|c|c|c|c|c|c|c|c|c|c|}
\hline $\begin{array}{c}\text { First } \\
\text { Author/Year/ } \\
\text { Region } \\
\text { (Country) }\end{array}$ & $\begin{array}{l}\text { Design } \\
\text { (Study } \\
\text { Type) }\end{array}$ & $\begin{array}{l}\text { Length of } \\
\text { Follow-Up } \\
\text { (Weeks) }\end{array}$ & $\begin{array}{l}\text { Target } \\
\text { Population } \\
\text { (Age) }\end{array}$ & Inclusion Criteria & Exclusion Criteria & $\begin{array}{c}\text { Sample Size at } \\
\text { Baseline (Total } \\
n / \text { Female }(\%))\end{array}$ & Assessment Tool & $\begin{array}{c}\text { Target } \\
\text { Lifestyles }\end{array}$ & $\begin{array}{l}\text { Sample Size at } \\
\text { Follow-Up } \\
\text { (Total } n / \text { Female } \\
(\%))\end{array}$ & $\begin{array}{l}\text { Statistical } \\
\text { Technique/s }\end{array}$ \\
\hline $\begin{array}{c}\text { Caruso, } \\
\text { (2020) } \\
\text { Bari (Italy) }\end{array}$ & RS & $\begin{array}{c}2 \text { weeks } \\
\text { before and } \\
2 \text { weeks } \\
\text { after } \\
\text { lockdown }\end{array}$ & $\begin{array}{l}\text { People } \geq 18 \\
\text { years old with } \\
\text { a diagnose of } \\
\text { type } 1 \text { diabetes } \\
\text { mellitus }\end{array}$ & $\begin{array}{l}\text { Both males and } \\
\text { females older than } \\
18 \text { years old } \\
\text { diagnosed with } \\
\text { diabetes mellitus } \\
\text { type } 1 \text { for at least } 1 \\
\text { year. Being on an } \\
\text { intensive insulin } \\
\text { regimen for at least } 6 \\
\text { months and Having } \\
\text { their glucose data } \\
\text { uploaded to the } \\
\text { Libreview platform }\end{array}$ & $\begin{array}{c}\text { History of acute } \\
\text { myocardial } \\
\text { infarction, stroke } \\
\text { and percutaneous or } \\
\text { surgical } \\
\text { revascularization } \\
\text { within the last } 30 \\
\text { days. (2) Active } \\
\text { neoplasia within the } \\
\text { last } 6 \text { months and (3) } \\
\text { Any other } \\
\text { concomitant medical } \\
\text { or psychological } \\
\text { condition }\end{array}$ & $\begin{array}{c}n=48 \\
\text { Female }=47.9 \% \\
(n=23)\end{array}$ & $\begin{array}{c}\text { Changes in dietary } \\
\text { habits were assessed } \\
\text { on a } \\
\text { semi-quantitative } \\
\text { basis } \\
\text { (higher/lower/same } \\
\text { frequency) with an } \\
\text { ad hoc questionnaire }\end{array}$ & $\begin{array}{l}\text { Physical activity } \\
\text { and diet }\end{array}$ & $\begin{array}{c}n=48 \\
\text { Female }=47.9 \% \\
(n=23)\end{array}$ & $\begin{array}{l}\text { (1) Two-tailed paired } \\
\text { Student's } t \text {-test (2) } \\
\text { Wilcoxon } \\
\text { matched-pairs } \\
\text { signed-rank test, } \\
\text { and (3) chi-square } \\
\text { test }\end{array}$ \\
\hline $\begin{array}{l}\text { Pellegrini, } \\
\text { (2020) } \\
\text { Nothern Italy } \\
\text { (Italy) }\end{array}$ & RS & 4 weeks & $\begin{array}{l}\text { Patients aged } \\
18-75 \text { years old }\end{array}$ & $\begin{array}{l}\text { Patients of the } \\
\text { Obesity Unit of the } \\
\text { Diabetes and } \\
\text { Metabolic Diseases } \\
\text { Clinic of the Città } \\
\text { dela Salute e della } \\
\text { Scienza Hospital of } \\
\text { Torino }\end{array}$ & Dropouts & $\begin{array}{c}n=150 \\
\text { Female }=77.33 \% \\
(n=116)\end{array}$ & $\begin{array}{l}\text { A 12-question } \\
\text { multiple-choice } \\
\text { questionnaire }\end{array}$ & $\begin{array}{l}\text { Physical activity } \\
\text { and diet }\end{array}$ & $\begin{array}{c}n=150 \\
\text { Female }=77.33 \% \\
(n=116)\end{array}$ & $\begin{array}{c}\text { (1) } T \text {-test for paired } \\
\text { samples, (2) } \\
\text { Mann-Whitney test } \\
(2 \text { groups), (3) } \\
\text { Kruskal-Wallis test } \\
\text { (3 groups), (4) } \\
\text { multiple regression } \\
\text { model, and (6) } \\
\text { regression path } \\
\text { model }\end{array}$ \\
\hline $\begin{array}{l}\text { Martínez- } \\
\text { Steele, (2020) } \\
\text { All regions } \\
\text { (Brazil) }\end{array}$ & PS & 15 weeks & $\begin{array}{l}\text { People } \geq 18 \\
\text { years old }\end{array}$ & $\begin{array}{c}\text { People older than } 18 \\
\text { years old residing in } \\
\text { Brazil }\end{array}$ & No & $\begin{array}{c}n=10,116 \\
\text { Female }=78 \% \\
\quad(n=7895)\end{array}$ & $\begin{array}{c}\text { A simplified } \\
\text { questionnaire about } \\
\text { diet }\end{array}$ & Diet & $\begin{array}{c}n=10,116 \\
\text { Female }=78 \% \\
(n=7895)\end{array}$ & $\begin{array}{l}\text { (1) Chi square, and } \\
\text { (2) } t \text {-test }\end{array}$ \\
\hline
\end{tabular}


Table 3. Cont.

\begin{tabular}{|c|c|c|c|c|c|c|c|c|c|c|}
\hline $\begin{array}{c}\text { First } \\
\text { Author/Year/ } \\
\text { Region } \\
\text { (Country) }\end{array}$ & $\begin{array}{l}\text { Design } \\
\text { (Study } \\
\text { Type) }\end{array}$ & $\begin{array}{l}\text { Length of } \\
\text { Follow-Up } \\
\text { (Weeks) }\end{array}$ & $\begin{array}{l}\text { Target } \\
\text { Population } \\
\text { (Age) }\end{array}$ & Inclusion Criteria & Exclusion Criteria & $\begin{array}{l}\text { Sample Size at } \\
\text { Baseline (Total } \\
n / \text { Female }(\%))\end{array}$ & Assessment Tool & $\begin{array}{c}\text { Target } \\
\text { Lifestyles }\end{array}$ & $\begin{array}{c}\text { Sample Size at } \\
\text { Follow-Up } \\
\text { (Total } n / \text { Female } \\
(\%))\end{array}$ & $\begin{array}{l}\text { Statistical } \\
\text { Technique/s }\end{array}$ \\
\hline $\begin{array}{l}\text { Deschasaux- } \\
\text { Tanguy. } \\
(2020) \\
\text { All regions } \\
\text { (France) }\end{array}$ & PS & 24 weeks & $\begin{array}{l}\text { French people } \\
\geq 18 \text { years old }\end{array}$ & $\begin{array}{c}\text { Adults aged } \geq 18 \\
\text { years old living in } \\
\text { France }\end{array}$ & No & $\begin{array}{c}n=37,252 \\
\text { Female }=52.3 \% \\
(n=19.483)\end{array}$ & $\begin{array}{l}\text { Web-based 24-h } \\
\text { dietary records }\end{array}$ & $\begin{array}{l}\text { Food-related } \\
\text { practices, } \\
\text { physical } \\
\text { activity, and } \\
\text { body weight }\end{array}$ & $\begin{array}{c}n=37,252 \\
\text { Female }=52.3 \% \\
(n=19.483)\end{array}$ & $\begin{array}{l}\text { (1) The Alternative } \\
\text { Healthy Eating } \\
\text { Index (AHEI)-2010 } \\
\text { score, (2) NOVA } \\
\text { classification, (3) } \\
\text { multiple } \\
\text { correspondence } \\
\text { analysis, (4) } \\
\text { ANCOVA models } \\
\text { for variations in } \\
\text { continuous } \\
\text { variables, and (5) } \\
\text { multivariable } \\
\text { logistic regression } \\
\text { models }\end{array}$ \\
\hline $\begin{array}{l}\text { Zhang (2020) } \\
\text { All regions } \\
\text { (China) }\end{array}$ & RS & 20 weeks & $\begin{array}{l}\text { Adults living } \\
\text { in China aged } \\
\text { 18-80 years old }\end{array}$ & $\begin{array}{l}\text { Adults } 18-80 \text { years } \\
\text { old living in } \\
\text { mainland China }\end{array}$ & No & $\begin{array}{c}n=1994 \\
\text { Female }=62.79 \% \\
(n=1252)\end{array}$ & $\begin{array}{l}\text { (1) A standardized } \\
\text { survey, (2) the } \\
\text { Household Dietary } \\
\text { Diversity Score } \\
\text { (HDDS), and (3) a } \\
\text { series of questions } \\
\text { regarding changes in } \\
\text { dietary behaviours }\end{array}$ & Diet & $\begin{array}{c}\quad n=1994 \\
\text { Female }=62.79 \% \\
\quad(n=1252)\end{array}$ & $\begin{array}{l}\text { (1) Univariate } \\
\text { analysis, (2) A linear } \\
\text { regression model, (3) } \\
\text { K-means clustering } \\
\text { analysis, and (4) } \\
\text { Multivariable } \\
\text { analysis }\end{array}$ \\
\hline $\begin{array}{l}\text { Yu. (2020) } \\
\text { All regions } \\
\text { (China) }\end{array}$ & RS & 4 weeks & $\begin{array}{l}\text { People from } \\
\text { China aged } \\
17-22 \text { years old }\end{array}$ & $\begin{array}{l}\text { Youth people under } \\
\text { three educational } \\
\text { attachments (i.e., } \\
\text { high school, college } \\
\text { or graduate) in } \\
\text { China }\end{array}$ & No & $\begin{array}{c}n=10,082 \\
\text { Female }=72.39 \% \\
(n=7298)\end{array}$ & $\begin{array}{l}\text { COVID-19 Impact } \\
\text { on Lifestyle Change } \\
\text { Survey (COINLICS) }\end{array}$ & Diet & $\begin{array}{c}n=10,082 \\
\text { Female }=72.39 \% \\
(n=7298)\end{array}$ & $\begin{array}{l}\text { (1) Descriptive } \\
\text { statistics to } \\
\text { summarise } \\
\text { characteristics of } \\
\text { participants, (2) Pair } \\
\text { t-test to compare the } \\
\text { differences in } \\
\text { frequency of food } \\
\text { and beverage } \\
\text { consumption, and } \\
\text { (3) A factor analysis } \\
\text { with orthogonal } \\
\text { rotation to derive } \\
\text { main dietary } \\
\text { patterns before-after } \\
\text { the pandemic }\end{array}$ \\
\hline
\end{tabular}


Table 3. Cont

\begin{tabular}{|c|c|c|c|c|c|c|c|c|c|c|}
\hline $\begin{array}{c}\text { First } \\
\text { Author/Year/ } \\
\text { Region } \\
\text { (Country) } \\
\end{array}$ & $\begin{array}{l}\text { Design } \\
\text { (Study } \\
\text { Type) }\end{array}$ & $\begin{array}{l}\text { Length of } \\
\text { Follow-Up } \\
\text { (Weeks) }\end{array}$ & $\begin{array}{l}\text { Target } \\
\text { Population } \\
\text { (Age) }\end{array}$ & Inclusion Criteria & Exclusion Criteria & $\begin{array}{l}\text { Sample Size at } \\
\text { Baseline (Total } \\
n / \text { Female }(\%))\end{array}$ & Assessment Tool & $\begin{array}{c}\text { Target } \\
\text { Lifestyles }\end{array}$ & $\begin{array}{l}\text { Sample Size at } \\
\text { Follow-Up } \\
\text { (Total } n / \text { Female } \\
(\%))\end{array}$ & $\begin{array}{l}\text { Statistical } \\
\text { Technique/s }\end{array}$ \\
\hline $\begin{array}{c}\text { Barone, } \\
\text { (2021) } \\
\text { Pennsylvania } \\
\text { (USA) }\end{array}$ & PS & 54 weeks & $\begin{array}{l}\text { Desk workers } \\
\geq 18 \text { years old }\end{array}$ & $\begin{array}{l}\text { Participants in the } \\
\text { RESET BP trial }\end{array}$ & No & $\begin{array}{c}n=112 \\
\text { Female }=69 \% \\
(n=77)\end{array}$ & $\begin{array}{l}\text { Diet Screener } \\
\text { Questionnaire }\end{array}$ & $\begin{array}{c}\text { Sedentary } \\
\text { behaviour, } \\
\text { physical } \\
\text { activity, sleep } \\
\text { quality, and diet }\end{array}$ & $\begin{array}{c}n=112 \\
\text { Female }=69 \% \\
(n=77)\end{array}$ & $\begin{array}{c}\text { (1) Paired samples, } \\
\text { (2) McNemar tests, } \\
\text { and (3) Bowker's } \\
\text { tests }\end{array}$ \\
\hline $\begin{array}{l}\text { Curtis, (2021) } \\
\text { Adelaide } \\
\text { (Australia) }\end{array}$ & PS & 54 weeks & $\begin{array}{l}\text { Healthy adults } \\
\text { aged } 18-65 \\
\text { years old }\end{array}$ & $\begin{array}{l}\text { Adults residing in } \\
\text { greater metropolitan } \\
\text { Adelaide, having } \\
\text { access to a } \\
\text { Bluetooth-enabled } \\
\text { mobile device or } \\
\text { computer and home } \\
\text { internet, proficiency } \\
\text { in English, and } \\
\text { ambulant }\end{array}$ & $\begin{array}{l}\text { Pregnancy, having } \\
\text { an implanted } \\
\text { electronical medical } \\
\text { device, or } \\
\text { experiencing or } \\
\text { receiving treatment } \\
\text { for any } \\
\text { life-threatening } \\
\text { condition impacting } \\
\text { daily lifestyle and } \\
\text { health }\end{array}$ & $\begin{array}{c}n=61 \\
\text { Female }=65.6 \% \\
(n=40)\end{array}$ & $\begin{array}{c}\text { Dietary } \\
\text { Questionnaire for } \\
\text { Epidemiological } \\
\text { Studies (DQUES and } \\
\text { diet v3.2; Cancer } \\
\text { Council Victoria) }\end{array}$ & $\begin{array}{l}\text { Physical } \\
\text { activity, and } \\
\text { diet }\end{array}$ & $\begin{array}{c}n=61 \\
\text { Female }=65.6 \% \\
(n=40)\end{array}$ & $\begin{array}{l}\text { Mixed effects } \\
\text { models }\end{array}$ \\
\hline $\begin{array}{l}\text { Jia, (2021) } \\
\text { All regions } \\
\text { (China) }\end{array}$ & RS & 20 weeks & $\begin{array}{l}\text { Chinese youth } \\
\text { aged } 15-28 \\
\text { years old }\end{array}$ & No & No & $\begin{array}{c}n=10,082 \\
\text { female }=71.7 \% \\
(n=7320)\end{array}$ & $\begin{array}{l}\text { (1) Food frequency } \\
\text { questionnaire, and } \\
\text { (2) Beverages Diet } \\
\text { frequency } \\
\text { questionnaire }\end{array}$ & Diet & $\begin{array}{c}n=10,082 \\
\text { Female }=71.7 \% \\
(n=7320)\end{array}$ & $\begin{array}{l}\text { (1) Chi square, and } \\
\text { (2) T-test }\end{array}$ \\
\hline $\begin{array}{l}\text { Lamarche, } \\
\text { (2021) } \\
\text { Quebec } \\
\text { (Canada) }\end{array}$ & PS & 20 weeks & $\begin{array}{l}\text { Adults living } \\
\text { in Quebec } \geq 18 \\
\text { years old }\end{array}$ & $\begin{array}{l}\text { Adults aged } \geq 18 \\
\text { years old with a } \\
\text { residential address } \\
\text { in the province of } \\
\text { Quebec }\end{array}$ & $\begin{array}{l}\text { Not being able to } \\
\text { read and understand } \\
\text { French or English. } \\
\text { Not having access to } \\
\text { the Internet (with a } \\
\text { phone, tablet or } \\
\text { computer), and not } \\
\text { having an active } \\
\text { email address. } \\
\text { Living outside of the } \\
\text { province of Quebec }\end{array}$ & $\begin{array}{c}n=853 \\
\text { female }=87.2 \% \\
(n=744)\end{array}$ & $\begin{array}{l}\text { Self-administered } \\
\text { web-based } 24-h \text { time } \\
\text { dietary recall }\end{array}$ & $\begin{array}{l}\text { Smoking, } \\
\text { screen time, } \\
\text { physical } \\
\text { activity, and } \\
\text { diet }\end{array}$ & $\begin{array}{c}n=853 \\
\text { Female }=87.2 \% \\
(n=744)\end{array}$ & $\begin{array}{l}\text { Linear mixed } \\
\text { regression models }\end{array}$ \\
\hline $\begin{array}{l}\text { Czenczek- } \\
\text { Lewandowska. } \\
(2021) \\
\text { South-east } \\
\text { Poland } \\
\text { (Poland) }\end{array}$ & RS & 5 weeks & $\begin{array}{l}\text { Polish people } \\
\text { in early } \\
\text { adulthood } \\
\text { aged 18-34 } \\
\text { years old }\end{array}$ & $\begin{array}{l}\text { People aged from } 18 \\
\text { to } 34 \text { years of Polish } \\
\text { nationality resident } \\
\text { in south-east Poland } \\
\text { throughout the } \\
\text { pandemic period }\end{array}$ & No & $\begin{array}{c}n=506 \\
\text { Female }=70.2 \% \\
(n=355)\end{array}$ & $\begin{array}{c}\text { The modified Food } \\
\text { Frequency } \\
\text { Questionnaire } \\
\text { (FFQ-6) }\end{array}$ & $\begin{array}{l}\text { Diet, physical } \\
\text { activity and } \\
\text { sedentary } \\
\text { behaviour, and } \\
\text { sleep quality }\end{array}$ & $\begin{array}{c}n=506 \\
\text { Female }=70.2 \% \\
(n=355)\end{array}$ & \\
\hline
\end{tabular}


Table 3. Cont.

\begin{tabular}{|c|c|c|c|c|c|c|c|c|c|c|}
\hline $\begin{array}{c}\text { First } \\
\text { Author/Year/ } \\
\text { Region } \\
\text { (Country) } \\
\end{array}$ & $\begin{array}{l}\text { Design } \\
\text { (Study } \\
\text { Type) }\end{array}$ & $\begin{array}{l}\text { Length of } \\
\text { Follow-Up } \\
\text { (Weeks) }\end{array}$ & $\begin{array}{l}\text { Target } \\
\text { Population } \\
\text { (Age) }\end{array}$ & Inclusion Criteria & Exclusion Criteria & $\begin{array}{l}\text { Sample Size at } \\
\text { Baseline (Total } \\
n / \text { Female }(\%))\end{array}$ & Assessment Tool & $\begin{array}{c}\text { Target } \\
\text { Lifestyles }\end{array}$ & $\begin{array}{l}\text { Sample Size at } \\
\text { Follow-Up } \\
\text { (Total } n / \text { Female } \\
(\%))\end{array}$ & $\begin{array}{l}\text { Statistical } \\
\text { Technique/s }\end{array}$ \\
\hline $\begin{array}{l}\text { Naughton. } \\
(2021) \\
\text { High } \\
\text { deprivation } \\
\text { areas of all } \\
\text { regions } \\
\text { (United } \\
\text { Kigndom) }\end{array}$ & PS & 12 weeks & $\begin{array}{l}\text { Adult- } \\
\text { vulnerable } \\
\text { people from } \\
\text { the United } \\
\text { Kingdom } \geq 18 \\
\text { years old }\end{array}$ & $\begin{array}{l}\text { Adults living in the } \\
\text { United Kingdom. } \\
\text { Those with a } \\
\text { physical high-risk } \\
\text { health condition for } \\
\text { COVID-19 (in line } \\
\text { with the UK } \\
\text { National Health } \\
\text { Service definitions), } \\
\text { those living in a } \\
\text { high deprivation } \\
\text { area, and those with } \\
\text { a self-reported } \\
\text { mental health issue. }\end{array}$ & $\begin{array}{l}\text { People without } \\
\text { access to a } \\
\text { smartphone }\end{array}$ & $\begin{array}{c}n=1044 \\
\text { Female }=72.7 \% \\
(n=747) \\
* \text { Other }=0.2 \% \\
(n=2)\end{array}$ & $\begin{array}{l}\text { (1) An online survey, } \\
\text { and (2) Daily health } \\
\text { behaviour } \\
\text { monitoring }\end{array}$ & $\begin{array}{l}\text { Diet, physical } \\
\text { activity, alcohol, } \\
\text { smoking and } \\
\text { vaping, and } \\
\text { substance use } \\
\text { behaviours }\end{array}$ & $\begin{array}{c}n=1044 \\
\text { Female }=72.7 \% \\
(n=747) \\
* \text { Other }=0.2 \% \\
(n=2)\end{array}$ & $\begin{array}{l}\text { (1) Descriptive } \\
\text { analyses for all } \\
\text { health behaviour } \\
\text { measures, (2) } \\
\text { Multivariable } \\
\text { regression models, } \\
\text { and (3) Covariates }\end{array}$ \\
\hline $\begin{array}{l}\text { Imaz- } \\
\text { Aramburu. } \\
\text { (2021) } \\
\text { Basque } \\
\text { Country } \\
\text { (Spain) }\end{array}$ & PS & 48 weeks & $\begin{array}{l}\text { Students from } \\
\text { Spain } \geq 19 \\
\text { years old }\end{array}$ & $\begin{array}{l}\text { Nursing and } \\
\text { medical students at } \\
\text { the beginning of the } \\
\text { second year of their } \\
\text { degree and a } \\
\text { physiotherapy } \\
\text { student at the } \\
\text { beginning of the } \\
\text { third year at the } \\
\text { Faculty of Medicine } \\
\text { and Nursing, } \\
\text { University of the } \\
\text { Basque Country, } \\
\text { Spain }\end{array}$ & No & $\begin{array}{c}n=267 \\
\text { Female }=76 \% \\
(n=203)\end{array}$ & $\begin{array}{c}\text { (1) An ad hoc } \\
\text { self-administered } \\
\text { questionnaire, (2) } \\
\text { the Mediterranean } \\
\text { diet (MedDiet) } \\
\text { questionnaire }\end{array}$ & $\begin{array}{c}\text { Diet, and } \\
\text { physical activity }\end{array}$ & $\begin{array}{c}n=267 \\
\text { Female }=76 \% \\
(n=203)\end{array}$ & $\begin{array}{l}\text { (1) Kolmogorov- } \\
\text { Smirnov test, (2) a } \\
\text { Wilcoxon } \\
\text { signed-rank test to } \\
\text { analyze the } \\
\text { difference between } \\
\text { MedDiet scores, } \\
\text { moderate and } \\
\text { intense physical } \\
\text { activity, and eating } \\
\text { habits, (3) Paired } \\
t \text {-tests, and (4) a } \\
\text { McNemar test to } \\
\text { compare } \\
\text { proportions between } \\
\text { adherence to the } \\
\text { Mediterranean Diet } \\
\text { and physical activity } \\
\text { frequency }\end{array}$ \\
\hline $\begin{array}{l}\text { Segre. (2021) } \\
\text { Milan (Italy) }\end{array}$ & RS & 3 weeks & $\begin{array}{l}\text { Italian children } \\
\text { and } \\
\text { adolescents } \\
\text { aged 6-14 } \\
\text { years old }\end{array}$ & $\begin{array}{l}\text { Primary or middle } \\
\text { school students } \\
\text { living in Milan }\end{array}$ & $\begin{array}{l}\text { Not being able to } \\
\text { attend Zoom } \\
\text { Meetings }\end{array}$ & $\begin{array}{c}n=82 \\
\text { Female }=46.3 \% \\
(n=38)\end{array}$ & $\begin{array}{c}10-20 \\
\text { min-structured } \\
\text { interviews }\end{array}$ & $\begin{array}{c}\text { Distance } \\
\text { learning, eating, } \\
\text { and sleeping } \\
\text { habits }\end{array}$ & $\begin{array}{c}n=82 \\
\text { Female }=46.3 \% \\
(n=38)\end{array}$ & $\begin{array}{l}\text { Chi-square and } \\
\text { Fisher's exact test. }\end{array}$ \\
\hline
\end{tabular}


Table 3. Cont.

\begin{tabular}{|c|c|c|c|c|c|c|c|c|c|c|}
\hline $\begin{array}{c}\text { First } \\
\text { Author/Year/ } \\
\text { Region } \\
\text { (Country) } \\
\end{array}$ & $\begin{array}{l}\text { Design } \\
\text { (Study } \\
\text { Type) }\end{array}$ & $\begin{array}{l}\text { Length of } \\
\text { Follow-Up } \\
\text { (Weeks) }\end{array}$ & $\begin{array}{l}\text { Target } \\
\text { Population } \\
\text { (Age) }\end{array}$ & Inclusion Criteria & Exclusion Criteria & $\begin{array}{l}\text { Sample Size at } \\
\text { Baseline (Total } \\
n / \text { Female }(\%))\end{array}$ & Assessment Tool & $\begin{array}{c}\text { Target } \\
\text { Lifestyles }\end{array}$ & $\begin{array}{c}\text { Sample Size at } \\
\text { Follow-Up } \\
\text { (Total } n / \text { Female } \\
(\%))\end{array}$ & $\begin{array}{l}\text { Statistical } \\
\text { Technique/s }\end{array}$ \\
\hline $\begin{array}{l}\text { Maffoni. } \\
\text { (2021) } \\
\text { All regions } \\
\text { (Italy) }\end{array}$ & RS & 2 weeks & $\begin{array}{l}\text { Italian people } \\
\geq 18 \text { years old }\end{array}$ & $\begin{array}{l}\text { Adults }>18 \text { years } \\
\text { residing in Italy }\end{array}$ & No & $\begin{array}{c}n=1304 \\
\text { Female }=74.62 \% \\
(n=973)\end{array}$ & $\begin{array}{c}\text { (1) A } 38 \\
\text { multiple-choice } \\
\text { web-form survey in } \\
\text { Google Forms, and } \\
\text { (2) } 10 \\
\text { multiple-choice } \\
\text { items }\end{array}$ & $\begin{array}{l}\text { Water intake, } \\
\text { alcohol } \\
\text { consumption, } \\
\text { physical } \\
\text { activity, diet }\end{array}$ & $\begin{array}{c}n=1304 \\
\text { Female }=74.62 \% \\
(n=973)\end{array}$ & $\begin{array}{c}\text { (1) T-test, and (2) } \\
\text { Multiple Regression } \\
\text { Analyses }\end{array}$ \\
\hline $\begin{array}{l}\text { Hosomi. } \\
\text { (2021) } \\
\text { Kyoto } \\
\text { (Japan) }\end{array}$ & RS & 2 weeks & $\begin{array}{c}\text { Patients } \\
\text { diagnosed with } \\
\text { Type } 1 \text { Diabetes } \\
\text { Mellitus, aged } \\
\text { 16-75 years old }\end{array}$ & $\begin{array}{l}\text { Patients with Type } 1 \\
\text { Diabetes Mellitus } \\
\text { who visited the } \\
\text { Department of } \\
\text { Endocrinology and } \\
\text { Metabolism of the } \\
\text { Kyoto Prefectural } \\
\text { University of } \\
\text { Medicine }\end{array}$ & $\begin{array}{l}\text { Patients with } \\
\text { missing data }\end{array}$ & $\begin{array}{c}n=34 \\
\text { Female }=67.65 \% \\
(n=23)\end{array}$ & $\begin{array}{l}\text { A self-administered } \\
\text { questionnaire } \\
\text { related to stress and } \\
\text { lifestyle factors }\end{array}$ & $\begin{array}{l}\text { Stress levels, } \\
\text { sleep time, } \\
\text { exercise } \\
\text { intensity levels, } \\
\text { total diet, snack, } \\
\text { and prepared } \\
\text { food intake. }\end{array}$ & $\begin{array}{c}n=34 \\
\text { Female }=67.65 \% \\
(n=23)\end{array}$ & $\begin{array}{l}\text { (1) Descriptive } \\
\text { analyses, (2) } \\
\text { Spearman's } \\
\text { correlation, (3) and } \\
\text { Pair } t \text {-test to } \\
\text { evaluate differences } \\
\text { before and after the } \\
\text { pandemic }\end{array}$ \\
\hline $\begin{array}{l}\text { Herle. (2021) } \\
\text { All regions } \\
\text { (United } \\
\text { Kingdom) }\end{array}$ & PS & 5 weeks & $\begin{array}{l}\text { Adults } \geq 18 \\
\text { years old }\end{array}$ & $\begin{array}{l}\text { Adults living in the } \\
\text { UK during the } \\
\text { COVID-19 } \\
\text { pandemic }\end{array}$ & No & $\begin{array}{c}n=22,374 \\
\text { Female }=76 \% \\
(n=16,984)\end{array}$ & $\begin{array}{c}\text { Self-reported eating } \\
\text { changes }\end{array}$ & $\begin{array}{l}\text { Eating } \\
\text { behaviour, } \\
\text { mental health, } \\
\text { physical health } \\
\text { and body } \\
\text { weight }\end{array}$ & $\begin{array}{c}n=22,374 \\
\text { Female }=76 \% \\
(n=16,984)\end{array}$ & $\begin{array}{l}\text { (1) Descriptive } \\
\text { statistics, and (2) } \\
\text { Latent class growth } \\
\text { analysis to derive } \\
\text { trajectories of } \\
\text { change in eating } \\
\text { across lockdown }\end{array}$ \\
\hline $\begin{array}{l}\text { Dun. (2021) } \\
\text { Zhejiang and } \\
\text { Hunan } \\
\text { (China) }\end{array}$ & RS & 3 weeks & $\begin{array}{c}\text { Chinese } \\
\text { students aged } \\
17-27 \text { years old }\end{array}$ & $\begin{array}{c}\text { Students from } \\
\text { Hunan Traditional } \\
\text { Chinese Medical } \\
\text { College (Hunan, } \\
\text { China) and from the } \\
\text { Medical College of } \\
\text { Jinhua Polytechnic } \\
\text { (Zhejiang, China) } \\
\text { who were free of } \\
\text { chronic diseases and } \\
\text { had measured body } \\
\text { weight at the } \\
\text { universities before } \\
\text { the lockdown }\end{array}$ & No & $\begin{array}{c}n=12,889 \\
\text { Female }=80.22 \% \\
(n=10,340)\end{array}$ & $\begin{array}{l}\text { An 81-item-online } \\
\text { follow-up } \\
\text { questionnaire }\end{array}$ & $\begin{array}{l}\text { Perceptions of } \\
\text { COVID-19, } \\
\text { physical } \\
\text { activity, } \\
\text { sedentary time, } \\
\text { meal frequency, } \\
\text { alcohol } \\
\text { consumption, } \\
\text { and mental } \\
\text { health }\end{array}$ & $\begin{array}{c}\quad n=12,889 \\
\text { Female }=80.22 \% \\
\quad(n=10,340)\end{array}$ & $\begin{array}{l}\text { (1) Paired } t \text {-test and } \\
\text { Wilcoxon } \\
\text { signed-rank test, (2) } \\
\text { A baseline body } \\
\text { weight-adjusted } \\
\text { linear regression for } \\
\text { associations with } \\
\text { dietary habits, and } \\
\text { (3) A multivariate } \\
\text { linear regression }\end{array}$ \\
\hline
\end{tabular}


Table 3. Cont.

\begin{tabular}{|c|c|c|c|c|c|c|c|c|c|c|}
\hline $\begin{array}{c}\text { First } \\
\text { Author/Year/ } \\
\text { Region } \\
\text { (Country) }\end{array}$ & $\begin{array}{l}\text { Design } \\
\text { (Study } \\
\text { Type) }\end{array}$ & $\begin{array}{l}\text { Length of } \\
\text { Follow-Up } \\
\text { (Weeks) }\end{array}$ & $\begin{array}{l}\text { Target } \\
\text { Population } \\
\text { (Age) }\end{array}$ & Inclusion Criteria & Exclusion Criteria & $\begin{array}{c}\text { Sample Size at } \\
\text { Baseline (Total } \\
n / \text { Female }(\%))\end{array}$ & Assessment Tool & $\begin{array}{c}\text { Target } \\
\text { Lifestyles }\end{array}$ & $\begin{array}{c}\text { Sample Size at } \\
\text { Follow-Up } \\
\text { (Total } n / \text { Female } \\
(\%))\end{array}$ & $\begin{array}{l}\text { Statistical } \\
\text { Technique/s }\end{array}$ \\
\hline $\begin{array}{l}\text { Sato. (2021) } \\
\text { All regions } \\
\text { (Japan) }\end{array}$ & PS & 19 weeks & $\begin{array}{l}\text { Adults } \geq 18 \\
\text { years old }\end{array}$ & $\begin{array}{l}\text { Adults living in } \\
\text { Japan }\end{array}$ & No & $\begin{array}{c}n=5929 \\
\text { Female }=69 \% \\
(n=4087)\end{array}$ & $\begin{array}{l}\text { CALO mama health } \\
\text { app }\end{array}$ & $\begin{array}{c}\text { Diet and } \\
\text { physical activity }\end{array}$ & $\begin{array}{c}n=5929 \\
\text { Female }=69 \% \\
(n=4087)\end{array}$ & $\begin{array}{l}\text { (1) Generalized } \\
\text { linear mixed models, } \\
\text { (2) A Poisson } \\
\text { distribution was to } \\
\text { the count of intake } \\
\text { of the food groups, } \\
\text { and (3) A negative } \\
\text { binomial } \\
\text { distribution was to } \\
\text { the count of } \\
\text { drinking alcohol }\end{array}$ \\
\hline
\end{tabular}




\subsubsection{Country and Design of the Studies}

The twenty-three longitudinal studies included the following countries: India [26], Australia [27,28], Spain [29,30], Italy [31-35], Brazil [36], France [37], China [38-41], the United States [42], Canada [43], Poland [44], the United Kingdom [45,46] and Japan [47,48].

Twelve $[26-30,36,37,42,43,45,46,48] \quad(50 \%)$ were prospective studies, and eleven [31-35,38-41,44,47] (42.86\%) were retrospective studies. Additionally, the length of follow-ups varied between $2[32,35,47]$ and 54 weeks [28,42].

\subsubsection{Target Population}

Although studies mainly focused on adult populations over 18 years old with no comorbidities [28,35-38,42-44,46,48], specific subgroups that might be taken into account were found: people diagnosed with diabetes mellitus $[26,32,47]$, young people $[27,29-31,34,39-41]$, people with obesity [33] and people in vulnerable situations [45]. The average of mean ages for twenty-three longitudinal studies was 24.19 years $(\mathrm{SD}=15.3$ ).

\subsubsection{Sample Size}

Due to the differences among the studies, sample sizes varied between 34 [47] and 37,252 [37] participants (media $n=4918$; IQR 112 to 10,082). However, the experimental mortality was not significant except for one study [27], which lost almost $44.84 \%$ of the participants. In addition, the ratio between males and females was relatively equal, with the exception of five studies in which samples were comprised mainly of females $[27,33,36,41,43]$. This systematic review evaluated a total of 116,952 participants.

\subsubsection{Assessment Tools}

All the studies used different recording measures to assess target eating behaviour changes. In this sense, many of them were standardized validated scales, such as the NSW Centre for Public Health Nutrition [27], The Mediterranean Diet Quality Index for children and teenagers (KIDMED) [29], the 12-items lifestyle questionnaire [31], the House hold Dietary Diversity Score (HDDS) [38], the COVID-19 Impact on Lifestyle Change Survey (COINLICS) [39], the Diet Screener Questionnaire, the Dietary Questionnaire for Epidemiological Studies (DQUES and diet v3.2; Cancer Council Victoria) [28], the Food and Beverages Diet frequency questionnaire [40], the Modified Food frequency Questionnaire (FFQ-6) [44], and the Mediterranean diet (Med-Diet) questionnaire [30]; whereas the others used scales that were created for the specific purpose of their research [26,32-36,41,47]. Additionally, self-reports $[37,43,45,46]$ were used in order to follow eating changes among participants. One study used ecological momentary assessment through the CALO mama health app for this purpose [48].

\subsection{Objectives, Outcomes, and Results}

Table 4 summarizes the objectives, outcomes, and results of the studies, as shown below. 
Table 4. Objective, outcomes and results of the included studies.

\begin{tabular}{|c|c|c|c|}
\hline Authors & Objective & Outcome & Results \\
\hline Khare et al. (2020) & $\begin{array}{l}\text { To study the effect of lockdown on glycaemic control in } \\
\text { diabetic patients and possible factors responsible for this }\end{array}$ & Dietary changes (secondary outcome) & $\begin{array}{l}\text { Dietary changes were observed as a change in the type of diet, change } \\
\text { in timing of meals, change in frequency of meals and change in the } \\
\text { amount of diet. }\end{array}$ \\
\hline Medrano et al. (2020) & $\begin{array}{l}\text { To examine the effects of the COVID- } 19 \text { confinement on } \\
\text { lifestyle behaviours and to assess the influence of social } \\
\text { vulnerabilities on changes in lifestyle behaviours }\end{array}$ & $\begin{array}{l}\text { Adherence to the Mediterranean Diet } \\
\text { (primary outcome) }\end{array}$ & $\begin{array}{l}\text { Children worsened their lifestyle behaviours during the COVID-19 } \\
\text { confinement, specifically their adherence to the Mediterranean Diet. } \\
\text { No significant differences were found behaviours between primary } \\
\text { and secondary students. }\end{array}$ \\
\hline Pietrobelli et al. (2020) & $\begin{array}{l}\text { To test the hypothesis that factors contributing to weight gain } \\
\text { among children and adolescents with overweight and obesity } \\
\text { are exacerbated during a pandemic associated lockdown }\end{array}$ & $\begin{array}{l}\text { (1) Number of meals eaten per day, } \\
\text { (2) changes in vegetable and fruit intake } \\
\text { (3) potato chips intake, (4) red meat intake } \\
\text { and (5) sugary drinks } \\
\text { (primary outcomes) }\end{array}$ & $\begin{array}{c}\text { The number of meals eaten per day got increased. There were no } \\
\text { changes in vegetable intake, and fruit intake increased. There was an } \\
\text { increased consumptions of potato chips, red meat, and sugary drinks } \\
\text { during the lockdown. }\end{array}$ \\
\hline Caruso et al. (2020) & $\begin{array}{l}\text { To assess lockdown-related changes in glucose control and } \\
\text { variability and their association with psychological distress } \\
\text { and lifestyle changes in type } 1 \text { diabetes mellitus patients }\end{array}$ & $\begin{array}{l}\text { (1) Meals per day, (2) starchy foods, (3) sweets, } \\
\text { (4) whole grains and (5) vegetables } \\
\text { (secondary outcomes) }\end{array}$ & $\begin{array}{l}\text { Patients increased the frequency of starchy foods and sweets } \\
\text { consumption, respectively. }\end{array}$ \\
\hline Martínez-Steele et al. (2020) & $\begin{array}{c}\text { To describe the dietary characteristic of a cohort immediately } \\
\text { before and during the pandemic }\end{array}$ & $\begin{array}{c}\text { Changes in dietary characteristics (primary } \\
\text { outcome) }\end{array}$ & $\begin{array}{l}\text { The consumption of vegetables, fruits and legumes increased on daily } \\
\text { diet. There was a pattern of stability in the consumption of } \\
\text { ultra-processed food, although the number of people who consumed it } \\
\text { increased. }\end{array}$ \\
\hline Deschasaux-Tanguy. (2020) & $\begin{array}{c}\text { To explore potential changes in dietary intake, physical } \\
\text { activity, body weight and food supply during the COVID-19 } \\
\text { lockdown and how these differed according to individual } \\
\text { characteristics }\end{array}$ & $\begin{array}{l}\text { Determinants of nutrition-related } \\
\text { behaviour (primary outcome) }\end{array}$ & $\begin{array}{l}\text { Diet-related practices during COVID-19 lockdown were modified. } \\
\text { Trouble to keep a regular mealtime schedule, more frequent snaking } \\
\text { (at least } 3 \text { times a day, every day) and a decreased consumption of } \\
\text { fresh food was reported. Ultra-processed food consumption increased. }\end{array}$ \\
\hline Zhang. (2020) & $\begin{array}{l}\text { To explore post-lockdown dietary behaviours and their effects } \\
\text { on dietary diversity. }\end{array}$ & Dietary behaviour (primary outcome) & $\begin{array}{l}\text { An increased consumption of seafood and dietary supplements was } \\
\text { found. Also, frozen food and raw food consumption decreased, } \\
\text { whereas a higher alcohol and vinegar intake was registered. }\end{array}$ \\
\hline Yu. (2020) & $\begin{array}{c}\text { To assess changes in dietary patterns among youths in China } \\
\text { after COVID-19 lockdown }\end{array}$ & Dietary patterns (primary outcome) & $\begin{array}{l}\text { Significant changes in the weekly frequency of major food intake after } \\
\text { lockdown was reported. There was a decrease in rice intake while an } \\
\text { increase consumption of other staple food such as fish, eggs, fresh } \\
\text { vegetables, preserved vegetables, fresh fruit and dairy products was } \\
\text { recorded. There was an increased consumption of wheat products and } \\
\text { a decrease in the frequency of beverages intake. }\end{array}$ \\
\hline
\end{tabular}


Table 4. Cont

\section{Authors}

Barone. (2021)

Curtis. (2021)

Jia. (2021)

re changes in diet patterns during the COVID-19 lockdown among youths in China

To study the longitudinal impact of COVID-19 on work practices, lifestyle and well-being among desk workers during COVID-19 lockdown

To examine changes in activity patterns, recreational physical activities, diet, weight and wellbeing from before to during COVID-19 restrictions

Diet patterns (primary outcome)

Dietary habits (primary outcome)

Dietary intake (primary outcome) energy from protein and a greater percentage of energy from alcohol were recorded.

Significant changes in diet patterns associated with more frequent consumption of wheat products, other staple foods, preserved vegetables, and tea and less frequent consumption of rice, meat, poultry, fresh vegetables and fruit, soybean and dairy products and sugar-sweetened beverages were found.

There were small but significant increases in the following components: whole grains, greens and beans, refined grains (reduced consumption), total vegetables, total dairy, seafood and plant proteins, added sugar (reduced consumption), and total proteins. The overall diet quality slightly improved.

To document the change in diet quality and in food insecurit
observed during the COVID-19-related early lockdown

Diet quality (primary outcome)

To assess whether and to what extent the outbreak of the
COVID-19 pandemic influenced the health-promoting
(2021)

(2021) activity, sedentary behaviours and sleep.

Eating habits (primary outcome)

Average consumption of sweets, snacks, cereal products increased. A significant higher intake of alcohol and fats was recorded.

There were reductions in the mean reported number of daily portions of vegetables and fruit but no change in reported portions of high sugar food consumed. In the case of alcohol consumption, there was an increase in the average intake per month.

Naughton. (2021)

To provide baseline cohort descriptives and assess change in

Dietary behaviour (primary outcome)

There was a greater adherence to the Mediterranean Diet. The consumption of vegetables increased significantly during the

To understand the influence that the pandemic exerted on the Imaz-Aramburu. (2021) health sciences programs
hity students, in particular

Eating habits (secondary outcome) An increased consumption of nuts was recorded.

The amount of food eaten increased, with a higher consumption of junk food, snacks and sweets.

Milan, one of the Italian cities most affected by COVID-19

Eating habits (primary outcome)

To investigate lifestyle habits and eating behaviours

Maffoni. (2021) modifications in a sample of Italian adults during "Phase 1" COVID-19 pandemic home confinement

Eating behaviours (primary outcome)

Negative changes in eating behaviour were found. These included an increased consumption of desserts or sweets at lunch. Craving or eating between meals was reported as well as the higher consumption of unhealthy snacks, beverages and ultra-processed food.

To investigate the effects of the COVID-19 pandemic on the
glycaemic control and changes in stress levels and lifestyle in glycaemic control and changes in stress leve
patients with T1D.
Diet (primary outcome)
Average amount of food intake got increased. The consumption of snacks and prepared food were also considerably increased. 
Table 4. Cont.

Authors

\section{Objective}

To describe how people's eating behaviour (eating more, (eating more, lockdown in the UK

Herle. (2021)

To observe weight change in Chinese youth during a 4-month

lockdown and the associations between weight change and

COVID-19-related stress, anxiety, depression, physical activity, sedentary time and dietary habits.

Dun. (2021)

To study dietary changes during the pandemic while considering changes in work and life patterns
Sato. (2021)

Outcome

Results

Eating behaviour (primary outcome)

Many individuals did experience changes to their food intake as well as lower adherence to healthy eating patterns

Dietary habits (secondary outcome)

Dietary changes (primary outcome)
A higher alcohol consumption was found in particular among men. Daily snack frequency also increased. more meats and alcohol compared with non-workers. In particular,

rregular workers consumed less vegetables, mushrooms and fish. The average daily number of self-made meals increased among all participants. 


\subsubsection{Objectives}

All of the studies had a common objective, assessing eating behaviour changes caused by the COVID-19 pandemic. In this sense, sixteen studies focused on eating behaviour changes during lockdown conditions $[26,29,31-35,37-43,45,46]$, while seven studies focused their research into the entire spectrum of the pandemic $[27,28,30,36,44,47,48]$.

\subsubsection{Outcomes}

Out of twenty-three studies, eighteen studies pursued eating behaviour as a primary or main outcome $[27,28,32-40,42-48]$, while five studies included it as a secondary outcome [26,29-31,41].

\subsubsection{Results}

All of the studies recorded eating behaviour changes during the COVID-19 pandemic in comparison to previous baselines before the pandemic, concluding that there were changes in the type of diet. Two studies found that there were changes in mealtimes [26,37], whereas three reported a more frequent food intake $[26,35,39]$ and four an increased amount of food eaten $[26,31,46,47]$. In particular, six studies highlighted a greater consumption of snacks [33-35,41,44,47]. In this sense, five studies established that there was a preference for sweets [32-35,44] while two concluded that wheat products were more frequently consume after the COVID-19 pandemic [39,40]. In the case of beverages, two studies found a higher daily intake $[35,40]$ while another one reported a decreased intake [39]. Specifically, for alcohol, six studies reported an increased consumption [28,38,41,44,45,48].

Otherwise, four studies reported a decreased consumption of fruit and vegetables [39, $40,45,48]$, two that there were no substantial changes [27,31], and two that the daily intake got increased $[30,36]$. Regarding this, three studies found a decreased consumption of fresh food $[37,39,40]$ and four other studies reported a higher intake of ultra-processed food [35-37,47]. Meat consumption got decreased according to two studies [40,42], but also got increased regarding three studies $[30,31,48]$.

Finally, three studies resolved that negative changes in eating behaviour were found due to a lower adherence to healthy diets $[29,35,46]$, meanwhile two reported adherence to healthy diet $[30,43]$ and one study declared no changes in dietary habits just except for a reduction in the amount of meat eaten [42].

\subsection{Quality of Studies}

To assess the quality of the studies, the Newcastle-Ottawa Quality Assessment Scale (NOS) [49] was used. This tool was developed with the aim of being helpful for systematic reviews so that quality criteria can be reached while ensuring a low risk of bias. NOS uses three main categories: selection, comparability and outcome. Each category contains several items about the characteristics of observational studies, each of which includes several answers. Among those answers, at least one is accompanied by a star (经). As stars symbolize a low risk of bias, once the scale is completed, it is necessary to sum them. If the result is less than 7 stars, there might be a high risk of bias. Additionally, in the case of reviewers selecting answers that do not include a star, it is important to check the possible biases of the study. Therefore, NOS scores might be categorized into three groups: very high risk of bias (0-3 stars), high risk of bias (4-6 stars) and low risk of bias (7-9 stars) [50].

The quality of each study was evaluated independently by two reviewers. A third reviewer was assigned in case of disagreement. The final consensus is shown in Table 5. 
Table 5. Quality appraisal studies.

\begin{tabular}{|c|c|c|c|c|c|c|c|c|c|c|}
\hline \multirow{2}{*}{ Study ID } & \multicolumn{3}{|c|}{ Selection } & \multicolumn{3}{|c|}{ Comparability } & \multicolumn{2}{|l|}{ Outcome } & \multirow[b]{2}{*}{ Total } & \multirow[b]{2}{*}{ Score } \\
\hline & 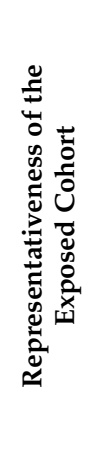 & 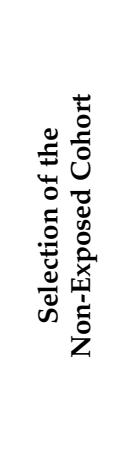 & 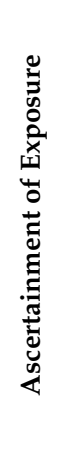 & 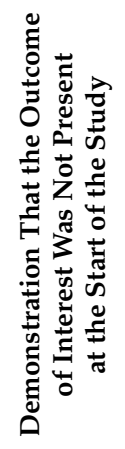 & 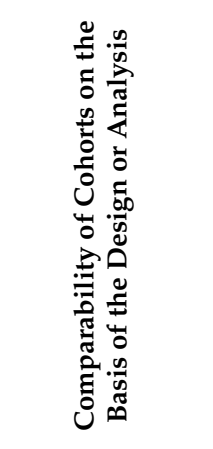 & 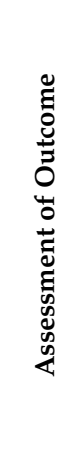 & 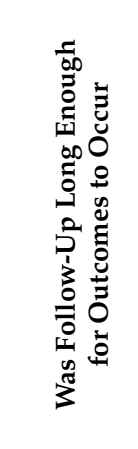 & 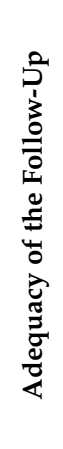 & & \\
\hline Khare et al. (2020) & is & 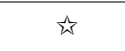 & 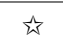 & 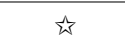 & 论的 & is & is & 站 & 9 & Low risk of bias \\
\hline Munasinghe et al. (2020) & 站 & 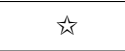 & 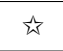 & & 放 & 放 & 放 & 访 & 7 & Low risk of bias \\
\hline Caruso et al. (2020) & $\hbar$ & 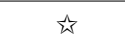 & 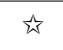 & 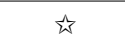 & 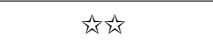 & 放弥 & & 就 & 9 & Low risk of bias \\
\hline Medrano et al. (2020) & 站 & 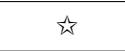 & 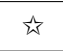 & 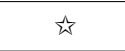 & 放 & 旅实 & 站 & 站 & 9 & Low risk of bias \\
\hline Pellegrini et al. (2020) & is & 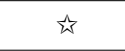 & $\sqrt{2}$ & th & th & 㧒 & 放 & 㧒 & 8 & Low risk of bias \\
\hline Martínez-Steele et al. (2020) & 放 & 放 & $\sqrt{2}$ & 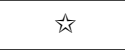 & 放 & 放 & is & 放 & 8 & Low risk of bias \\
\hline Deschasaux-Tanguy et al. (2020) & th & $\vec{s}$ & & 糸 & th & 序 & 放 & 糸 & 7 & Low risk of bias \\
\hline Zhang et al. (2020) & 约 & 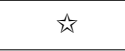 & 放 & is & 㧒 & 旅它 & 放 & it & 9 & Low risk of bias \\
\hline Yu et al. (2020) & 放 & $\sqrt{5}$ & $\sqrt{5}$ & 站 & 放 & 站 & 放 & & 7 & Low risk of bias \\
\hline Barone et al. (2021) & 站 & 弐 & $\sqrt{5}$ & th & 糸 & 施序 & 放 & & 8 & Low risk of bias \\
\hline Curtis et al. (2021) & 㧒 & 约 & & 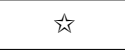 & 㧒 & 放㑔 & is & 约 & 8 & Low risk of bias \\
\hline Jia et al. (2021) & 放 & $\sqrt{5}$ & & & 放 & 放㑔 & th & $\sqrt{2}$ & 7 & Low risk of bias \\
\hline Lamarche et al. (2021) & 弐 & $\sqrt{3}$ & $\sqrt{2}$ & 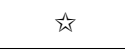 & 㶦 & 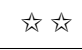 & 放 & 访 & 9 & Low risk of bias \\
\hline Czenczek-Lewandowska et al. (2021) & 弐 & $\sqrt{2}$ & $\sqrt{3}$ & $\sqrt{2}$ & 放 & 站 & 放 & & 7 & Low risk of bias \\
\hline Naughton et al. (2021) & $\vec{t}$ & $\sqrt{2}$ & $\sqrt{5}$ & $\sqrt{5}$ & 放 & 旅为 & 放 & 放 & 9 & Low risk of bias \\
\hline Imaz-Aramburu et al. (2021) & 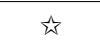 & & 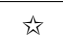 & 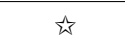 & 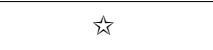 & 就数 & 站 & 站 & 8 & Low risk of bias \\
\hline Segre et al. (2021) & th & 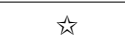 & is & 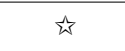 & 站 & it & is & & 7 & Low risk of bias \\
\hline Maffoni et al. (2021) & 站 & is & & 放 & is & is & 放 & 站 & 7 & Low risk of bias \\
\hline
\end{tabular}


Table 5. Cont.

\begin{tabular}{|c|c|c|c|c|c|c|c|c|c|c|}
\hline & & Selection & & & Comparability & & Outcome & & & \\
\hline Study ID & 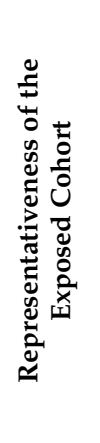 & 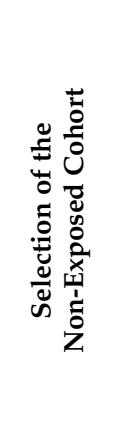 & 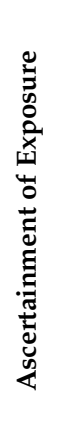 & 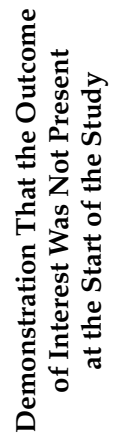 & 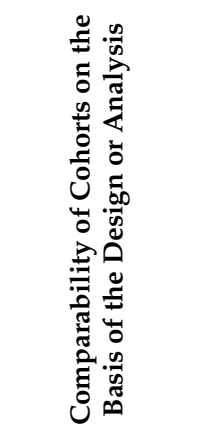 & 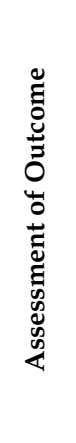 & 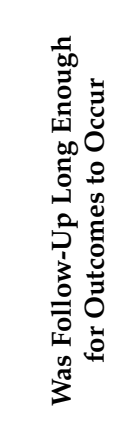 & 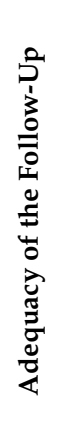 & Total & Score \\
\hline Hosomi et al. (2021) & 必 & 必 & 为 & 必 & 必 & 氺 & 次 & & 7 & Low risk of bias \\
\hline Herle et al. (2021) & ts & 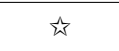 & & 放 & $\hat{x}$ & 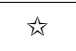 & is & $\hat{s}$ & 7 & Low risk of bias \\
\hline Dun et al. (2021) & 斥 & 为 & 头 & 为 & 为 & 场 & & 场 & 7 & Low risk of bias \\
\hline Sato et al. (2021) & 必 & $\hbar$ & & 必 & 幽 & 不动 & th & 地 & 8 & Low risk of bias \\
\hline
\end{tabular}

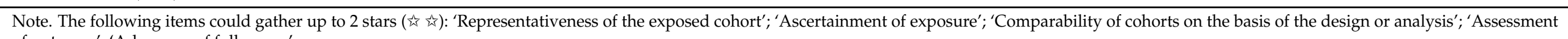
of outcome'; 'Adequacy of follow-up'. 
To quantify the degree of agreement between reviewers regarding overall quality assessment, the Cohen Kappa Index was calculated. The results showed that there was a good agreement level (Cohen Kappa Index $=0.74 ; 95 \%$ CI, 0.60 to 0.89 ) regarding the specific score of each study. By considering this, it was possible to conclude that there was a low risk of bias in all twenty-three studies.

\section{Discussion}

The current systematic review, which included twenty-three studies, provides a comprehensive overview of eating behaviour characteristics associated with the COVID-19 pandemic. Consistent with previous reviews, several outcomes, such as overeating $[26,31,46,47]$ and the influence of personal preferences on food choices [32-35,44], have been observed. However, this systematic review also found that the frequency of meals is not associated with the amount of food eaten $[32,36,40]$. Additionally, variables, such as gender and age, that did not show a correlation with specific eating behaviours [29], need to be considered, as well as the influence of mental health $[41,45,46]$. In the case of work status, one study found that people who spend more time at home consumed a greater amount of self-made food [48] something that appears to be correlated with the availability of food delivery services [51], which are a faster option for people who is in a rush.

However, when referring to population with no comorbidities, results may generate controversy. Three studies reported patterns of stability on eating behaviour despite the outbreak of the pandemic $[27,28,42]$, while two studies even reported improvements in the adherence to healthy diets $[38,43]$. In spite of that, results showed a more frequent intake of food, an increased consumption of ultra-processed food and a higher caloric intake due to a more frequent alcohol consumption [35,37,44,46,48].

Regarding specific subgroups, people with diabetes appeared to increase the daily amount of food eaten. In this sense, unhealthy food products such as sweets and starchy food were common among this population [26,32,47]. People with obesity showed similar trends by reporting a significant increase in the amount and frequency of unhealthy food products [33]. In the case of young people, a lower adherence to healthy diets such as the Mediterranean Diet [29] was found due to an increased intake of food, a preference for snacks and a lack of fruit and vegetables intake [27,29-31,34,39-41]. On the contrary, vulnerable population showed a reduced intake of food, contrasting with an increased alcohol consumption [45]. In all these cases, results did not show changes towards a healthier diet, what may emphasize the need to protect vulnerable population from risk situations that might affect health or, in this case, nutritional status.

By considering all this information, it is possible to confirm the existence of changes in eating behaviour during the COVID-19 pandemic. To the best of our knowledge, this is the first systematic review that examined these trends by considering longitudinal studies. With this design, it is possible to analyse the changes of eating behaviour in comparison with previous baselines before the pandemic, which made it possible to establish the level of significance of this phenomenon. Additionally, the screening process that was applied ensured the quality of this review, as shown in the NOS results of each study. Therefore, it might be possible to accept the relevance of this review on this topic.

Even so, several limitations should be considered when interpreting these results. First, due to the heterogeneity of the designs as well as the small set of papers used, it is important to consider this review as a first approach to eating behaviour changes during the COVID-19 pandemic. Its results may be useful to consider when undertaking future directions of reviews when more studies become available. Second, and according to the above, due to the novelty of this phenomenon, there were no comparators apart from within-subject comparability (prospective and retrospective), which is a limitation when trying to distinguish between the influence of the pandemic (lockdowns, social distance, isolation, uncertainty about the future, etc.) and any other external factor. Longitudinal between-subject studies may be necessary to dismiss possible bias caused by external factors. Third, this systematic review included both clinical and non-clinical population 
what may disturb results due to additional variables that has not been measured. Consequently, any interpretation should be made carefully, as the effect size might not represent society-wide eating behaviour, although previous studies have established common eating behaviour changes among clinical and non-clinical populations [21,22]. Finally, although one of the strengths of this review is that different countries have been included, it might be important to consider that restrictive measures have been different among countries so that the COVID-19 pandemic might have had a greater or lesser impact depending on the policies of each country [36].

Keeping all of this in mind, a deep understanding beyond the results is still possible. Therefore, this review is useful as a benchmark that contributes to the current body of knowledge about the impact of the COVID-19 pandemic on daily lifestyles. In addition to confirming the appearance of changes in eating behaviour since the outbreak of the COVID19 pandemic, this is the first systematic review that has achieved an accurate description of those changes by considering different countries. The results of this study will provide a reference to guide future research directions among those interested not only in this topic but also in specific eating patterns as well as in the differences between 'amount of food' and 'snack frequency'. All of these approaches will lead to a better understanding of eating behaviour during the COVID-19 pandemic as well as contribute to future guidelines about health promotion.

\section{Implications for Government Policies in Nutrition}

Due to the importance of eating behaviour as criteria on health and safety, it is important for governments to ensure healthy eating patterns among population through health education programmes and suitable access to food supply [52]. In this sense, nutritionsensitive policies might seek participation from different social and economic sectors with the aim of reaching a proper change in eating behaviour [53]. Some examples of these policies would include: (a) access to education, (b) the promotion of healthy local food environments as well as social protection programmes, (c) the regulation of working conditions, (d) the improvement of menus at school cantines and (e) the regulation of advertising through a gender-based perspective that rejects beauty standards and encourages a healthy lifestyle [54].

This initiative would address the Sustainable Development Goals (SDGs), in particular, the SDG 2 which aims to 'End hunger, achieve food security and improved nutrition and promote sustainable agriculture' [55]. Nutrition plays an important role within international cooperation as hunger elimination together with the development of sustainable food systems might improve several environmental aspects of strategic relevance and interest [56]. This systematic review provides important information about food choices in case of worldwide alarming situations such as the COVID-19 pandemic that might be responsible for a lack of food supply and negative emotions [4,9]. By considering that, future programmes aimed at mindful eating and healthy habits might include guidelines towards resources of interest during state alarms, examples of balanced diets and their budgets, area-based lists of soup kitchens, and allowances. Not only that but also, educational programmes might be extended beyond health and politic fields by applying multi-strategy campaigns concerned with sustained public awareness on healthy eating [53]. This kind of approach might be suitable for developed and developing countries as its objective would be ensuring a proper use of resources as well as healthier choices. Evidence-based interventions have demonstrated the efficacy of nutritional education programmes into adaptative eating behaviour [57], what might involve an urgent call to action for governments and social sectors focused on health promotion. Some implications of this initiative would include a lower demand of healthcare resources [58], a greater productivity in the workplace [59], and a better quality of life among countries due to the access to basic needs [60]. 


\section{Conclusions}

The COVID-19 outbreak led to changes in eating behaviour, which may have become less healthy during the pandemic. Although these changes could be a result of uncertainty and discomfort, adverse effects on health, especially for vulnerable population, would emphasize the need to promote healthy habits through preventive interventions and social actions supported by governments. Additionally, with the aim of assessing a more accurate framework of the stages through which eating behaviour changes evolved during this period, further research should be carried out. In this context, it would be important to focus on food intake but also on alcohol consumption and its consequences. By this, not only may a progression of 'eating behaviour during a state of alarm' be developed but also a benchmark for future directions can be established that will help improve guidelines for achieving proper nutrition aimed at the new normality.

Author Contributions: Conceptualization, C.G.-M., C.M.O.-S. and E.M.; methodology, C.G.-M., E.M. and I.G.-G.; formal analysis, C.G.-M., C.M.O.-S., I.G.-G. and E.M.; investigation, C.G.-M. and E.M.; writing—original draft preparation, C.G.-M.; writing—review and editing, C.G.-M. and E.M.; supervision, I.G.-G. and C.M.O.-S. All authors have read and agreed to the published version of the manuscript.

Funding: Emma Motrico and Irene Gómez-Gómez have received funding from Instituto de Salud Carlos III (PI19/01264) and Junta de Andalucia (PY20 RE 025 LOYOLA).

Institutional Review Board Statement: Not aplicable.

Informed Consent Statement: Not aplicable.

Data Availability Statement: Not aplicable.

Conflicts of Interest: The authors declare no conflict of interest.

\section{References}

1. Pérez Abreu, M.R.; Gómez Tejeda, J.J.; Dieguez Guach, R.A. Características clínico epidemiológicas de la COVID-19. Rev. Haban. Cienc. Méd. 2020, 19, e3254.

2. Negrini, S.; Grabljevec, K.; Boldrini, P.; Kiekens, C.; Moslavac, S.; Zampolini, M.; Christodoulou, N. Up to 2.2 million people experiencing disability suffer collateral damage each day of COVID-19 lockdown in Europe. Eur. J. Phys. Rehabil. Med. 2020, 56, 361-365. [CrossRef]

3. Parrado-González, A.; León-Jariego, J.C. Factores asociados al malestar emocional y morbilidad psíquica en población española [COVID-19: Factors associated with emotional distress and psychological morbidity in spanish population]. Rev. Esp. Salud Pública 2020, 94, e202006058.

4. Ammar, A.; Brach, M.; Trabelsi, K.; Chtourou, H.; Boukhris, O.; Masmoudi, L.; Bouaziz, B.; Bentlage, E.; How, D.; Ahmed, M.; et al. Effects of COVID-19 Home Confinement on Eating Behaviour and Physical Activity: Results of the ECLB-COVID19 International Online Survey. Nutrients 2020, 12, 1583. [CrossRef]

5. Balanzá-Martínez, V.; Atienza-Carbonell, B.; Kapczinski, F.; De Boni, R.B. Lifestyle behaviours during the COVID-19-Time to connect. Acta Psychiatr. Scand. 2020, 141, 399-400. [CrossRef]

6. Campbell, H.; Wood, A.C. Challenges in Feeding Children Posed by the COVID-19 Pandemic: A Systematic Review of Changes in Dietary Intake Combined with a Dietitian's Perspective. Curr. Nutr. Rep. 2021, 10, 155-165. [CrossRef]

7. Abu Hatab, A.; Krautscheid, L.; Boqvist, S. COVID-19, livestock systems and food security in developing countries: A systematic review of an emerging literature. Pathogens 2021, 10, 586. [CrossRef]

8. Scarmozzino, F.; Visioli, F. COVID-19 and the subsequent lockdown modified dietary habits of almost half the population in an Italian sample. Foods 2020, 9, 675. [CrossRef]

9. Mason, T.B.; Heron, K.E.; Braitman, A.L.; Lewis, R.J. A daily diary study of perceived social isolation, dietary restraint, and negative affect in binge eating. Appetite 2016, 97, 94-100. [CrossRef]

10. Moynihan, A.B.; van Tilburg, W.A.P.; Igou, E.R.; Wisman, A.; Donnelly, A.E.; Mulcaire, J.B. Eaten up by boredom: Consuming food to escape awareness of the bored self. Front. Psychol. 2015, 6, 369. [CrossRef]

11. Rolland, B.; Haesebaert, F.; Zante, E.; Benyamina, A.; Haesebaert, J.; Franck, N. Global changes and factors of increase in caloric/salty food intake, screen use, and substance use during the early COVID-19 containment phase in the general population in France: Survey study. JMIR Public Health Surveill. 2020, 6, e19630. [CrossRef]

12. Rothman, A.J.; Sheeran, P.; Wood, W. Reflective and automatic processes in the initiation and maintenance of dietary change. Ann. Behav. Med. 2009, 38 (Suppl. 1), S4-S17. [CrossRef] 
13. Zupo, R.; Castellana, F.; Sardone, R.; Sila, A.; Giagulli, V.A.; Triggiani, V.; Cincione, R.I.; Giannelli, G.; De Pergola, G. Preliminary trajectories in dietary behaviors during the COVID-19 pandemic: A public health call to action to face obesity. Int. J. Environ. Res. Public Health 2020, 17, 7073. [CrossRef]

14. Ruiz-Roso, M.B.; Padilha, P.d.C.; Mantilla-Escalante, D.C.; Ulloa, N.; Brun, P.; Acevedo-Correa, D.; Peres, W.A.F.; Martorell, M.; Aires, M.T.; Cardoso, L.d.O.; et al. Confinamiento del COVID-19 y cambios en las tendencias alimentarias de los adolescentes en Italia, España, Chile, Colombia y Brasil. Nutrients 2020, 12, 1807. [CrossRef]

15. Laguna, L.; Fiszman, S.; Puerta, P.; Chaya, C.; Tárrega, A. The impact of COVID-19 lockdown on food priorities. Results from a preliminary study using social media and an online survey with Spanish consumers. Food Qual. Prefer. 2020, 86, 104028. [CrossRef]

16. Sidor, A.; Rzymski, P. Dietary choices and habits during COVID-19 lockdown: Experience from Poland. Nutrients 2020, $12,1657$. [CrossRef] [PubMed]

17. Di Renzo, L.; Gualtieri, P.; Cinelli, G.; Bigioni, G.; Soldati, L.; Attinà, A.; Bianco, F.F.; Caparello, G.; Camodeca, V.; Carrano, E.; et al. Psychological aspects and eating habits during COVID-19 home confinement: Results of ehlc-COVID-19 italian online survey. Nutrients 2020, 12, 2152. [CrossRef]

18. Hutton, G.B.; Brugulat-panés, A.; Bhagtani, D.; Maadjhou, C.M. A Systematic Scoping Review of the Impacts of Community Food Production Initiatives in Kenya, Cameroon, and South Africa. J. Glob. Health Rep. 2021, 5, e2021010. [PubMed]

19. Chew, H.S.J.; Lopez, V. Global impact of COVID-19 on weight and weight-related behaviors in the adult population: A scoping review. Int. J. Environ. Res. Public Health 2021, 18, 1876. [CrossRef] [PubMed]

20. Neira, C.; Godinho, R.; Rincón, F.; Mardones, R.; Pedroso, J. Consequences of the COVID-19 syndemic for nutritional health: A systematic review. Nutrients 2021, 13, 1168. [CrossRef] [PubMed]

21. Damayanthi, H.D.W.T.; Prabani, K.I.P. Nutritional determinants and COVID-19 outcomes of older patients with COVID-19: A systematic review. Arch. Gerontol. Geriatr. 2021, 95, 104411. [CrossRef]

22. Huang, Y.; Yao, L.; Huang, Y.-M.; Wang, M.; Ling, W.; Sui, Y.; Zhao, H.-L. Obesity in patients with COVID-19: A systematic review and meta-analysis. Metab. Clin. Exp. 2020, 113, 154378. [CrossRef] [PubMed]

23. Moher, D.; Liberati, A.; Tetzlaff, J.; Altman, D.G.; Group, T.P. Preferred reporting items for systematic reviews and meta-analyses: The PRISMA statement. PLoS Med. 2009, 6, e1000097. [CrossRef]

24. Higgins, J.P.T.; Green, S. Cochrane Handbook for Systematic Reviews of Interventions Version 5.1.0; The Cochrane Collaboration: London, UK, 2011; pp. 197-255.

25. McHugh, M.L. Interrater reliability: The kappa statistic. Biochemia medica. Biochem. Medica 2012, 22, 276-282. [CrossRef]

26. Khare, J.; Jindal, S. Observational study on Effect of Lock Down due to COVID 19 on glycemic control in patients with Diabetes: Experience from Central India. Diabetes Metab. Syndr. Clin. Res. Rev. 2020, 14, 1571-1574. [CrossRef]

27. Munasinghe, S.; Sperandei, S.; Freebairn, L.; Conroy, E.; Jani, H.; Marjanovic, S.; Page, A. The Impact of Physical Distancing Policies During the COVID-19 Pandemic on Health and Well-Being Among Australian Adolescents. J. Adolesc. Health 2020, 67, 653-661. [CrossRef]

28. Curtis, R.G.; Olds, T.; Ferguson, T.; Fraysse, F.; Dumuid, D.; Esterman, A.; Hendrie, G.A.; Brown, W.J.; Lagiseti, R.; Maher, C.A. Changes in diet, activity, weight, and wellbeing of parents during COVID-19 lockdown. PLoS ONE 2021, 16, e0248008. [CrossRef] [PubMed]

29. Medrano, M.; Cadenas-Sanchez, C.; Oses, M.; Arenaza, L.; Amasene, M.; Labayen, I. Changes in lifestyle behaviours during the COVID-19 confinement in Spanish children: A longitudinal analysis from the MUGI project. Pediatr. Obes. 2021, 16, e12731. [CrossRef] [PubMed]

30. Imaz-Aramburu, I.; Fraile-Bermúdez, A.B.; Martín-Gamboa, B.S.; Cepeda-Miguel, S.; Doncel-García, B.; Fernandez-Atutxa, A.; Irazusta, A.; Zarrazquin, I. Influence of the COVID-19 pandemic on the lifestyles of health sciences university students in spain: A longitudinal study. Nutrients 2021, 13, 1958. [CrossRef]

31. Pietrobelli, A.; Pecoraro, L.; Ferruzzi, A.; Heo, M.; Faith, M.; Zoller, T.; Antoniazzi, F.; Piacentini, G.; Fearnbach, S.N.; Heymsfield, S.B. Effects of COVID-19 Lockdown on Lifestyle Behaviors in Children with Obesity Living in Verona, Italy: A Longitudinal Study. Obesity 2020, 28, 1382-1385. [CrossRef]

32. Caruso, I.; Di Molfetta, S.; Guarini, F.; Giordano, F.; Cignarelli, A.; Natalicchio, A.; Perrini, S.; Leonardini, A.; Giorgino, F.; Laviola, L. Reduction of hypoglycaemia, lifestyle modifications and psychological distress during lockdown following SARS-CoV-2 outbreak in type 1 diabetes. Diabetes. Metab. Res. Rev. 2020, 37, e3404.

33. Pellegrini, M.; Ponzo, V.; Rosato, R.; Scumaci, E.; Goitre, I.; Benso, A.; Belcastro, S.; Crespi, C.; De Michieli, F.; Ghigo, E.; et al. Changes in weight and nutritional habits in adults with obesity during the "lockdown" period caused by the COVID-19 virus emergency. Nutrients 2020, 12, 2016. [CrossRef]

34. Segre, G.; Campi, R.; Scarpellini, F.; Clavenna, A.; Zanetti, M.; Cartabia, M.; Bonati, M. Interviewing children: The impact of the COVID-19 quarantine on children's perceived psychological distress and changes in routine. BMC Pediatr. 2021, 21, 231. [CrossRef]

35. Maffoni, S.; Brazzo, S.; De Giuseppe, R.; Biino, G.; Vietti, I.; Pallavicini, C.; Cena, H. Lifestyle changes and body mass index during COVID-19 pandemic lockdown: An Italian online-survey. Nutrients 2021, 13, 1117. [CrossRef] [PubMed] 
36. Martínez Steele, E.; Rauber, F.; dos Santos Costa, C.; Leite, M.A.; Gabe, K.T.; da Costa Louzada, M.L.; Levy, R.B.; Monteiro, C.A. Dietary changes in the NutriNet Brasil cohort during the COVID-19 pandemic. Rev. Saude Publica 2020, 54, 91. [CrossRef] [PubMed]

37. Deschasaux-Tanguy, M.; Druesne-Pecollo, N.; Esseddik, Y.; De Edelenyi, F.S.; Allès, B.; Andreeva, V.A.; Baudry, J.; Charreire, H.; Deschamps, V.; Egnell, M.; et al. Diet and physical activity during the coronavirus disease 2019 (COVID-19) lockdown (March-May 2020): Results from the French NutriNet-Santé cohort study. Am. J. Clin. Nutr. 2021, 113, 924-938. [CrossRef] [PubMed]

38. Zhang, J.; Zhao, A.; Ke, Y.; Huo, S.; Ma, Y.; Zhang, Y.; Ren, Z.; Li, Z.; Liu, K. Dietary behaviors in the post-lockdown period and its effects on dietary diversity: The second stage of a nutrition survey in a longitudinal chinese study in the COVID-19 era. Nutrients 2020, 12, 3269. [CrossRef] [PubMed]

39. Yu, B.; Zhang, D.; Yu, W.; Luo, M.; Yang, S.; Jia, P. Impacts of lockdown on dietary patterns among youths in China: The COVID-19 Impact on Lifestyle Change Survey. Public Health Nutr. 2021, 24, 3221-3232. [CrossRef]

40. Jia, P.; Liu, L.; Xie, X.; Yuan, C.; Chen, H.; Guo, B.; Zhou, J.; Yang, S. Changes in dietary patterns among youths in China during COVID-19 epidemic: The COVID-19 impact on lifestyle change survey (COINLICS). Appetite 2021, 158, 105015. [CrossRef]

41. Dun, Y.; Ripley-Gonzalez, J.W.; Zhou, N.; You, B.; Li, Q.; Li, H.; Zhang, W.; Thomas, R.J.; Olson, T.P.; Liu, J.; et al. Weight gain in Chinese youth during a 4-month COVID-19 lockdown: A retrospective observational study. BMJ Open 2021, 11, e052451. [CrossRef]

42. Barone Gibbs, B.; Kline, C.E.; Huber, K.A.; Paley, J.L.; Perera, S. COVID-19 shelter-at-home and work, lifestyle and well-being in desk workers. Occup. Med. 2021, 71, 86-94. [CrossRef]

43. Lamarche, B.; Brassard, D.; Lapointe, A.; Laramée, C.; Kearney, M.; Côté, M.; Bélanger-Gravel, A.; Desroches, S.; Lemieux, S.; Plante, C. Changes in diet quality and food security among adults during the COVID-19-related early lockdown: Results from NutriQuébec. Am. J. Clin. Nutr. 2021, 113, 984-992. [CrossRef]

44. Czenczek-Lewandowska, E.; Wyszyńska, J.; Leszczak, J.; Baran, J.; Weres, A.; Mazur, A.; Lewandowski, B. Health behaviours of young adults during the outbreak of the COVID-19 pandemic-A longitudinal study. BMC Public Health 2021, 21, 1038. [CrossRef] [PubMed]

45. Naughton, F.; Ward, E.; Khondoker, M.; Belderson, P.; Marie Minihane, A.; Dainty, J.; Hanson, S.; Holland, R.; Brown, T.; Notley, C. Health behaviour change during the UK COVID-19 lockdown: Findings from the first wave of the C-19 health behaviour and well-being daily tracker study. Br. J. Health Psychol. 2021, 26, 624-643. [CrossRef] [PubMed]

46. Herle, M.; Smith, A.D.; Bu, F.; Steptoe, A.; Fancourt, D. Trajectories of eating behavior during COVID-19 lockdown: Longitudinal analyses of 22,374 adults. Clin. Nutr. ESPEN 2021, 42, 158-165. [CrossRef]

47. Hosomi, Y.; Munekawa, C.; Hashimoto, Y.; Okamura, T.; Takahashi, F.; Kawano, R.; Nakajima, H.; Majima, S.; Senmaru, T.; Nakanishi, N.; et al. The effect of COVID-19 pandemic on the lifestyle and glycemic control in patients with type 1 diabetes: A retrospective cohort study. Diabetol. Int. 2021, 1-6. [CrossRef]

48. Sato, K.; Kobayashi, S.; Yamaguchi, M.; Sakata, R.; Sasaki, Y.; Murayama, C.; Kondo, N. Working from home and dietary changes during the COVID-19 pandemic: A longitudinal study of health app (CALO mama) users. Appetite 2021, 165, 105323. [CrossRef]

49. Wells, G.; Brodsky, L.; O'Connell, D.; Shea, B.; Henry, D.; Mayank, S.; Tugwell, P. An evaluation of the Newcastle Ottawa Scale: An assessment tool for evaluating the quality of non-randomized studies. XI Cochrane Colloq. Evid. Health Care Cult. 2003, 26-31. Available online: https:/ / cmr.cochrane.org/?CRGReportID=5100 (accessed on 1 October 2021).

50. Lo, C.K.L.; Mertz, D.; Loeb, M. Newcastle-Ottawa Scale: Comparing reviewers' to authors' assessments. BMC Med. Res. Methodol. 2014, 14, 45. [CrossRef] [PubMed]

51. Skotnicka, M.; Karwowska, K.; Kłobukowski, F.; Wasilewska, E.; Małgorzewicz, S. Dietary Habits before and during the COVID-19 Epidemic inSelected European Countries. Nutrients 2021, 1690, 1690. [CrossRef] [PubMed]

52. Alsaffar, A.A. Sustainable diets: The interaction between food industry, nutrition, health and the environment. Food Sci. Technol. Int. 2016, 22, 102-111. [CrossRef]

53. Mozaffarian, D.; Angell, S.Y.; Lang, T.; Rivera, J.A. Role of government policy in nutrition-barriers to and opportunities for healthier eating. BMJ 2018, 361, k2426. [CrossRef] [PubMed]

54. Friel, S.; Hattersley, L.; Ford, L. Evidence Review: Addressing the Social Determinants of Inequities in Mental Wellbeing of Children and Adolescents; National Center for Epidemiology and Population Health: Canberra, ACT, Australia, 2013; Volume 62.

55. Grosso, G.; Mateo, A.; Rangelov, N.; Buzeti, T.; Birt, C. Nutrition in the context of the Sustainable Development Goals. Eur. J. Public Health 2020, 30, I19-I23. [CrossRef] [PubMed]

56. Meybeck, A.; Gitz, V. Conference on "Sustainable food consumption" Sustainable diets within sustainable food systems. Proc. Nutr. Soc. 2017, 76, 1-11. [CrossRef]

57. Warren, J.M.; Smith, N.; Ashwell, M. A structured literature review on the role of mindfulness, mindful eating and intuitive eating in changing eating behaviours: Effectiveness and associated potential mechanisms. Nutr. Res. Rev. 2017, 30, 272-283. [CrossRef] [PubMed]

58. Nelson, M.E.; Hamm, M.W.; Hu, F.B.; Abrams, S.A.; Griffin, T.S. Alignment of healthy dietary patterns and environmental sustainability: A systematic review. Adv. Nutr. 2016, 7, 1005-1025. [CrossRef] 
59. Drewnowski, A. Impact of nutrition interventions and dietary nutrient density on productivity in the workplace. Nutr. Rev. 2020, 78, 215-224. [CrossRef]

60. Bérenger, V.; Verdier-Chouchane, A. Multidimensional Measures of Well-Being: Standard of Living and Quality of Life Across Countries. World Dev. 2007, 35, 1259-1276. [CrossRef] 